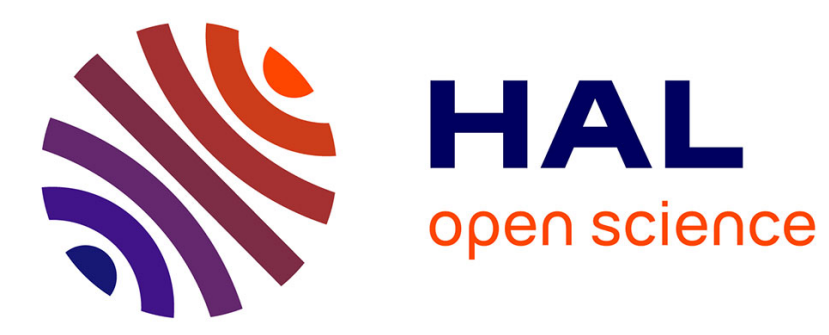

\title{
Two sided boundary stabilization of heterodirectional linear coupled hyperbolic PDEs
}

\author{
Jean Auriol, Florent Di Meglio
}

\section{To cite this version:}

Jean Auriol, Florent Di Meglio. Two sided boundary stabilization of heterodirectional linear coupled hyperbolic PDEs. IEEE Transactions on Automatic Control, inPress, 10.1109/TAC.2017.2763320 . hal-01318708v3

\section{HAL Id: hal-01318708}

\section{https://hal-mines-paristech.archives-ouvertes.fr/hal-01318708v3}

Submitted on 28 Nov 2016

HAL is a multi-disciplinary open access archive for the deposit and dissemination of scientific research documents, whether they are published or not. The documents may come from teaching and research institutions in France or abroad, or from public or private research centers.
L'archive ouverte pluridisciplinaire HAL, est destinée au dépôt et à la diffusion de documents scientifiques de niveau recherche, publiés ou non, émanant des établissements d'enseignement et de recherche français ou étrangers, des laboratoires publics ou privés. 


\title{
Two sided boundary stabilization of heterodirectional linear coupled hyperbolic PDEs
}

\author{
Jean Auriol ${ }^{1}$, and Florent Di Meglio ${ }^{1}$ \\ ${ }^{1}$ MINES ParisTech, PSL Research University, CAS - Centre automatique et systèmes, 60 bd St Michel 75006 Paris, France
}

\begin{abstract}
We solve the problem of stabilizing a general class of linear first-order hyperbolic systems using actuation at both boundaries of the spatial domain. We design a novel Fredholm transformation similarly to backstepping approaches to derive a boundary controller and a boundary observer enabling stabilization by output feedback. This yields an explicit full-state feedback law that achieves the theoretical lower bound for convergence to zero.
\end{abstract}

Index Terms-Distributed parameter systems, Linear systems, Backstepping, Stability of linear systems, Linear system observers

\section{INTRODUCTION}

$\mathbf{T}$ HIS article solves the problem of boundary stabilization of a general class of coupled heterodirectional linear first-order hyperbolic systems of Partial Differential Equations (PDEs) in minimum time, with arbitrary numbers $m$ and $n$ of PDEs in each direction. The actuation is applied on both boundaries.

Most physical systems involving a transport phenomenon can be modeled using hyperbolic partial differential equations (PDEs): traffic flow [4], heat exchangers [24], open channel flow [10], [13] or multiphase flow [15], [17]. The backstepping approach [18], [21] has enabled the design of stabilizing full-state feedback laws for these systems. These controllers are explicit, in the sense that they are expressed as a linear functional of the distributed state at each instant. The (distributed) gains can be computed offline. Comparing results obtained via backstepping design with existence results for stabilizing controllers reveals a gap. In [20], an extensive review of controllability results for linear hyperbolic systems is given, along with the theoretical lower bounds for convergence times. These bounds vary according, mainly, to the number and location of available actuators. Backstepping results have, until now, focused on single-boundary actuation, see e.g. [12] for the case of two coupled PDEs, [18] for an arbitrary number of PDEs or [5] for a minimum-time result in the general (single boundary actuation) case.

When actuation is applied at both boundaries, the literature usually focuses on design of dissipative boundary conditions to stabilize the system. This does not guarantee stabilization in the minimum theoretical time, and is only possible for small coupling terms between PDEs, but can generally be achieved using static boundary output feedback, which is much less computationally intensive. Recently the problem of stabilizing a system of two coupled PDEs with control at both sides has been solved in [22] in the case of reaction-diffusion PDEs and for 2-state heterodirectional linear PDEs with equal transport velocities. A generalization of this result for a general system of two hyperbolic PDEs is given in [6].

The main contribution of this paper is a generalization of this

Manuscript received December 1, 2012; revised August 26, 2015. Corresponding author: J. Auriol (email: jean.auriol@mines-paristech.fr). result with the design of a minimum time stabilizing controller in the general case of heterodirectional hyperbolic PDEs with actuation at both boundaries. A proposed boundary feedback law ensures finite-time convergence of the states to zero in minimum time. The minimum time defined [20] is the largest time between the different transport times in each direction.

Applications where controls and/or sensors are located at the two boundaries include control of open channel flow [7] and state and parameter estimation for oil drilling [14]. The process of drilling for hydrocarbons involves the circulation of a drilling fluid inside the serveral kilometer-long well to pressurize and clean the borehole. Under certain conditions, oil and gas flow from the reservoir into the well, resulting in a multiphase flow that needs to be monitored to ensure safety of operations. Sensors are typically located at the surface and, on more modern drilling facilities, at the bottom of the well. In this paper, we investigate the benefits of using sensors at both boundaries by conducting simulations on a distributed model for two-phase flow [2].

From a theoretical point of view, our approach, similar to the one presented in [6] is the following. Using a Fredholm transformation, the system is mapped to a target system with desirable stability properties. This target-system is designed as an exponentially stable cascade. The well-posedness of the Fredholm transformation is a consequence of a clever choice of the domain on which the kernels are defined and of the cascade structure of the target system. A full-state feedback law guaranteeing exponential stability of the zero equilibrium in the $\mathcal{L}^{2}$-norm is then designed. This full-state feedback law would require fully distributed measurements in practice, which is not realistic. For this reason we derive a boundary observer relying on measurements of the states at both boundaries.

The main technical difficulty of this paper is to prove wellposedness of the Fredholm transformation and its invertibility. Interestingly, the transformation kernels satisfy a system of equations with a cascade structure akin to the target system one. This structure enables a recursive proof of existence of the transformation kernels.

The paper is organized as follows. In Section II we introduce the model equations and the notations. In Section III we present the stabilization result: the target system and 
its properties are presented in Section III-A. In Section III$\mathrm{B}$, we derive the integral transformation and we present the domains on which the kernels are defined. The well-posedness of the kernel equations is proved in Section IV. This proof uses an important theorem which is given in Appendix in order to make the whole paper more readable. In Section $\mathrm{V}$ we prove the invertibility of the Fredholm transformation using an operator approach. The control feedback law and its properties are given in Section VI. In Section VII we present the uncollocated observer design. This observer is obtained with the a similar approach than the one developed for the control law. Finally, some simulation results are given in Section VIII. We conclude this article with some remarks presented in Section IX.

\section{Problem Description}

\section{A. System under consideration and notations}

We consider the following general linear hyperbolic system which appear in Saint-Venant equations, heat exchangers equations and other linear hyperbolic balance laws (see [8]).

$$
\begin{aligned}
& u_{t}(t, x)+\Lambda^{+} u_{x}(t, x)=\Sigma^{++} u(t, x)+\Sigma^{+-} v(t, x) \\
& v_{t}(t, x)-\Lambda^{-} v_{x}(t, x)=\Sigma^{-+} u(t, x)+\Sigma^{--} v(t, x)
\end{aligned}
$$

evolving in $\{(t, x) \mid t>0, \quad x \in[0,1]\}$, with the following linear boundary conditions

$$
u(t, 0)=U(t), \quad v(t, 1)=V(t)
$$

where

$$
\begin{gathered}
u=\left(u_{1} \ldots u_{n}\right)^{T}, \quad v=\left(v_{1} \ldots v_{m}\right)^{T} \\
\Lambda^{+}=\left(\begin{array}{ccc}
\lambda_{1} & & 0 \\
& \ddots & \\
0 & & \lambda_{n}
\end{array}\right), \quad \Lambda^{-}=\left(\begin{array}{ccc}
\mu_{1} & & 0 \\
& \ddots & \\
0 & & \mu_{m}
\end{array}\right)
\end{gathered}
$$

with constant speeds:

$$
-\mu_{m}<\cdots<-\mu_{1}<0<\lambda_{1}<\cdots<\lambda_{n}
$$

and constant real coupling matrices

$$
\begin{array}{ll}
\Sigma^{++}=\left\{\sigma_{i j}^{++}\right\}_{1 \leq i \leq n, 1 \leq j \leq n} & \Sigma^{+-}=\left\{\sigma_{i j}^{+-}\right\}_{1 \leq i \leq n, 1 \leq j \leq m} \\
\Sigma^{-+}=\left\{\sigma_{i j}^{-+}\right\}_{1 \leq i \leq m, 1 \leq j \leq n} & \Sigma^{--}=\left\{\sigma_{i j}^{--}\right\}_{1 \leq i \leq m, 1 \leq j \leq m}
\end{array}
$$

The initial conditions denoted $u_{0}$ and $v_{0}$ are assumed to belong to $L^{2}([0,1])$. In the following we denote

$$
\forall i, j \quad a_{i j}=\frac{\lambda_{i}}{\lambda_{i}+\mu_{j}}
$$

We also define $\bar{a}$ such that

$$
\left|\bar{a}-\frac{1}{2}\right|=\min _{1 \leq i \leq n, 1 \leq j \leq m}\left|a_{i j}-\frac{1}{2}\right|
$$

The $a_{i j}$ and $\bar{a}$ play an important role in the design of the target system.
Remark 1: $\bar{a}$ can be written as $a_{k l}$ with $k \in[0, n]$ and $l \in[0, m]$ (the uniqueness is not guaranteed). For this particular solution we denote $\bar{\lambda}$ and $\bar{\mu}$ the corresponding transport velocities.

Remark 2: The coupling terms are assumed constant here but the results of this paper can be adjusted for spatiallyvarying coupling terms.

Remark 3: Without any loss of generality we can assume that $\bar{a} \geq \frac{1}{2}$ (if this is not the case we make the change of variables $\bar{x}=1-x$ ). This assumption will be done in the following

\section{B. Well-posedness and operator formulation}

We can rewrite the system in the abstract form

$$
\frac{d}{d t}\left(\begin{array}{l}
u \\
v
\end{array}\right)=A\left(\begin{array}{l}
u \\
v
\end{array}\right)+B\left(\begin{array}{l}
U \\
V
\end{array}\right)
$$

where the operators $A$ and $B$ can be identified through their adjoints. The operator $A$ is thus defined by

$$
\begin{gathered}
A: D(A) \subset\left(L^{2}(0,1)\right)^{n+m} \rightarrow\left(L^{2}(0,1)\right)^{n+m} \\
\left(\begin{array}{l}
u \\
v
\end{array}\right) \longmapsto\left(\begin{array}{c}
-\Lambda^{+} u_{x}+\Sigma^{++} u+\Sigma^{+-} v \\
\Lambda^{-} v_{x}+\Sigma^{-+} u+\Sigma^{--} v
\end{array}\right)
\end{gathered}
$$

with

$$
D(A)=\left\{(u, v) \in\left(H^{1}(0,1)\right)^{n+m} \mid u(0)=v(1)=0\right\}
$$

A is well defined and its adjoint $A^{*}$ is

$$
\begin{aligned}
A^{*}: D\left(A^{*}\right) \subset\left(L^{2}(0,1)\right)^{n+m} \rightarrow\left(L^{2}(0,1)\right)^{n+m} \\
\left(\begin{array}{l}
u \\
v
\end{array}\right) \longmapsto\left(\begin{array}{c}
\Lambda^{+} u_{x}+\left(\Sigma^{++}\right)^{T} u+\left(\Sigma^{-+}\right)^{T} v \\
-\Lambda^{-} v_{x}+\left(\Sigma^{+-}\right)^{T} u+\left(\Sigma^{++}\right)^{T} v
\end{array}\right)
\end{aligned}
$$

with

$$
D\left(A^{*}\right)=\left\{(u, v) \in\left(H^{1}(0,1)\right)^{n+m} \mid u(1)=v(0)=0\right\}
$$

The operator $B$ is defined by

$$
<B\left(\begin{array}{l}
U \\
V
\end{array}\right),\left(\begin{array}{l}
z_{1} \\
z_{2}
\end{array}\right)>=z_{1}(0)^{t} \Lambda^{+} U+z_{2}(1)^{T} \Lambda^{-} V
$$

Its adjoint is

$$
B^{*}\left(\begin{array}{l}
z_{1} \\
z_{2}
\end{array}\right)=\left(\begin{array}{l}
z_{1}(0)^{T} \Lambda^{+} \\
z_{2}(1)^{T} \Lambda^{-}
\end{array}\right)
$$

\section{Control problem}

The goal is to design feedback control inputs $U(t)=$ $\left(U_{1}(t), \ldots, U_{n}(t)\right)^{T}$ and $V(t)=\left(V_{1}(t), \ldots, V_{m}(t)\right)^{T}$ such that the zero equilibrium is reached in minimum time $t=t_{F}$, where

$$
t_{F}=\max \left\{\frac{1}{\mu_{1}}, \frac{1}{\lambda_{1}}\right\}
$$

This "minimum time" is the time needed for the slowest characteristic to travel the entire length of the spatial domain. The existence of a control law reaching the null equilibrium in time $t_{F}$ is proved in [20] using a method of characteristics. To the best of our knowledge, no explicit feedback law has been designed to achieve this goal. Previous approaches yield 
- exponential stability for small coupling terms when twosided static output feedback is used [8].

- finite-time stability in time $\frac{1}{\lambda_{1}}+\frac{1}{\mu_{1}}>\max \left\{\frac{1}{\mu_{1}}, \frac{1}{\lambda_{1}}\right\}$ when one-sided backstepping design is used, i.e with one controlled boundary only ([5]).

- finite-time stability in time $\max \left\{\frac{1}{\mu}, \frac{1}{\lambda}\right\}$ when only two equations are considered ([6]).

In the second case, the system is mapped with a Volterra transformation to a target system that has a cascade structure, which is natural for backstepping. In the third case, a Fredholm transformation is used to map the system to a target system with desirable properties of stability. In the following we use a combination of these two approaches.

\section{CONTROL DESIGN}

The control design is based on a modified backstepping approach: using a specific transformation, we map the system (1)-(3) to a target system with desirable properties of stability. However, unlike the classical backstepping approach where a Volterra transformation is used, we use a Fredholm transformation here.

\section{A. Target system design}

We map the system (1)-(3) to the following system

$$
\begin{aligned}
\alpha_{t}(t, x)+\Lambda^{+} \alpha_{x}(t, x) & =\Omega(x) \alpha(t, x)+\Gamma(x) \beta(t, x) \\
\beta_{t}(t, x)-\Lambda^{-} \beta_{x}(t, x) & =\bar{\Omega}(x) \beta(t, x)+\bar{\Gamma}(x) \alpha(t, x)
\end{aligned}
$$

with the following boundary conditions

$$
\alpha(t, 0)=0 \quad \beta(t, 1)=0
$$

while $\Omega$ and $\bar{\Omega} \in L^{\infty}(0,1)$ are upper triangular matrices with the following structure

$$
\begin{array}{r}
\Omega(x)=\left(\begin{array}{cccc}
\omega_{1,1}(x) & \omega_{1,2}(x) & \ldots & \omega_{1, n}(x) \\
0 & \ddots & \ddots & \vdots \\
\vdots & \ddots & \omega_{n-1, n-1}(x) & \omega_{n-1, n}(x) \\
0 & \ldots & 0 & \omega_{n, n}(x)
\end{array}\right) \\
\bar{\Omega}(x)=\left(\begin{array}{cccc}
\bar{\omega}_{1,1}(x) & \bar{\omega}_{1,2}(x) & \ldots & \bar{\omega}_{1, m}(x) \\
0 & \ddots & \ddots & \vdots \\
\vdots & \ddots & \bar{\omega}_{m-1, m-1}(x) & \bar{\omega}_{m-1, m}(x) \\
0 & \ldots & 0 & \bar{\omega}_{m, m}(x)
\end{array}\right)
\end{array}
$$

The coefficients of the matrices $\Gamma(x)$ and $\bar{\Gamma}(x)$ are defined by

$$
\begin{aligned}
& \forall 1 \leq i \leq n \forall 1 \leq j \leq m \\
& \Gamma_{i j}(x)= \begin{cases}0 & \text { if } a_{i j} \geq \bar{a} \quad \text { or } \quad x<a_{i j} \\
\gamma_{i j}(x) & \text { otherwise }\end{cases}
\end{aligned}
$$

$$
\begin{aligned}
& \forall 1 \leq i \leq m \quad \forall 1 \leq j \leq n \\
& \bar{\Gamma}_{i j}(x)= \begin{cases}0 & \text { if } a_{j i}<1-\bar{a} \text { or } x>a_{j i} \\
\bar{\gamma}_{i j}(x) & \text { ohterwise }\end{cases}
\end{aligned}
$$

Remark 4: The $a_{i j}$ coefficients correspond to the spatial position where the characteristic leaving $x=0$ with velocity $\lambda_{i}$ and the one leaving $x=1$ with velocity $\mu_{j}$ intersect.

Remark 5: As it will appear in the proof, the coefficients $\Gamma$ and $\bar{\Gamma}$ do not make the system unstable due to their particular cascade structure. Their presence is necessary to prove the well-posedness of the backstepping transformation presented bellow. The following example illustrates this particular structure in a simple case.

Example 1: We consider the following coefficients

$$
n=3, m=2 \Lambda^{+}=\left(\begin{array}{ccc}
0.5 & 0 & 0 \\
0 & 2 & 0 \\
0 & 0 & 4
\end{array}\right) \Lambda^{-}=\left(\begin{array}{ll}
1 & 0 \\
0 & 3
\end{array}\right)
$$

We define the matrix $A$ such that $A_{i j}=a_{i j}=\frac{\lambda_{i}}{\lambda_{i}+\mu_{j}}$

$$
A=\left(\begin{array}{lll}
\frac{1}{3} & \frac{2}{3} & \frac{4}{5} \\
\frac{1}{7} & \frac{2}{5} & \frac{4}{7}
\end{array}\right)^{T}
$$

It yields $\bar{a}=a_{32}=\frac{4}{7}$. Consequently the matrices $\Gamma$ and $\bar{\Gamma}$ have the following structure

$$
\Gamma(x)=\left(\begin{array}{ll}
* & * \\
0 & * \\
0 & 0
\end{array}\right) \bar{\Gamma}(x)=\left(\begin{array}{ccc}
0 & * & * \\
0 & 0 & *
\end{array}\right)
$$

where the potential non-null terms are represented by $*$. These matrices have some structural properties that will be analyzed in Section IV-B.

Besides, the following lemma assesses the finite-time convergence of the target system.

Lemma 1: The system (19)-(20) reaches its zero equilibrium in finite-time $t_{F}=\max \left\{\frac{1}{\lambda}, \frac{1}{\mu}\right\}=\frac{1}{\lambda}$.

Proof: $\forall 1 \leq i \leq n, \forall 1 \leq j \leq n$, system (19)-(20) can be rewritten as

$$
\begin{aligned}
\alpha_{t}^{i}(t, x)+\lambda_{i} \alpha_{x}^{i}(t, x)= & \sum_{p=i}^{n} \omega_{i p}(x) \alpha^{p}(t, x) \\
& +\sum_{p=1}^{m} \gamma_{i p}(x) h_{\left[a_{i p}, 1\right]}(x) \beta^{p}(t, x) \\
\beta_{t}^{j}(t, x)-\mu_{j} \beta_{x}^{j}(t, x)= & \sum_{p=j}^{m} \bar{\omega}_{j p}(x) \beta^{p}(t, x) \\
& +\sum_{p=1}^{n} \bar{\gamma}_{j p}(x) h_{\left[0, a_{p j}\right]}(x) \alpha^{p}(t, x)
\end{aligned}
$$

where, for any interval $I, h_{I}$ is defined by

$$
h_{I}(x)= \begin{cases}1 & \text { if } x \in I \\ 0 & \text { else }\end{cases}
$$


with the convention

$$
\begin{array}{ll}
\gamma_{i j}(x)=0 & \text { if } \quad a_{i j}>\bar{a} \\
\bar{\gamma}_{i j}(x)=0 & \text { if } \quad a_{j i} \leq 1-\bar{a}
\end{array}
$$

By induction, let us consider the following property $P(s)$ defined for all $1 \leq s \leq n$

$P(s): \quad \forall p \geq n+1-s, \quad$ if $\quad t \geq \frac{x}{\lambda_{p}} \quad$ then $\quad \alpha^{p}(x, t)=0$

Initialization: The initialization can be proved using a similar technique than the one presented bellow in the induction and is not detailed here.

Induction: Let us assume that the property $P(s-1)(1<s \leq$ $n$ ) is true. We denote $r=n+1-s$ Integrating the $r^{t h}$ line of (29) along its characteristic lines and using the boundary condition $\alpha^{r}(t, 0)=0$, yields:

$$
\begin{aligned}
& \alpha^{r}(t, x)=\int_{0}^{\frac{x}{\lambda_{r}}} \sum_{p=r}^{n} \omega_{r p}\left(x-\lambda_{r} \nu\right) \alpha^{p}\left(t-\nu, x-\lambda_{r} \nu\right) \\
& +\sum_{p=1}^{m} \gamma_{r p}\left(x-\lambda_{r} \nu\right) h_{\left[a_{r p}, 1\right]}\left(x-\lambda_{r} \nu\right) \beta^{p}\left(t-\nu, x-\lambda_{r} \nu\right) d \nu
\end{aligned}
$$

with $x \in[0,1]$ and $t \geq \frac{x}{\lambda_{r}}$. Consequently, $\forall p>r$ :

$$
\begin{aligned}
t \geq \frac{x}{\lambda_{r}} & \Rightarrow\left(1-\frac{\lambda_{r}}{\lambda_{p}}\right) \frac{x}{\lambda_{r}} \leq t-\frac{x}{\lambda_{p}} \\
& \Rightarrow\left(1-\frac{\lambda_{r}}{\lambda_{p}}\right) \nu \leq t-\frac{x}{\lambda_{p}} \quad \forall \nu \in\left[0, \frac{x}{\lambda_{r}}\right] \\
& \Rightarrow t-\nu \geq \frac{x-\lambda_{r} \nu}{\lambda_{p}} \\
& \Rightarrow \alpha^{p}\left(t-\nu, x-\lambda_{r} \nu\right)=0
\end{aligned}
$$

The last implication uses the fact that $P(s-1)$ is true. Let us now consider the following property $P_{1}(q)$ defined for all $1 \leq q \leq m$

$$
P_{1}(q): \forall x>a_{r q}, \quad \forall t \geq \frac{1-x}{\mu_{q}}, \quad \beta^{q}(t, x)=0
$$

Initialization: The initialization can be proved using a similar technique than the one presented bellow in the induction and is not detailed here.

Induction: Let us assume that the property $P_{1}(q-1)(1<q \leq$ $m)$ is true. Integrating the $q^{\text {th }}$ line of (30) along its characteristic lines and using the boundary condition $\beta_{q}(t, 1)=0$, yields:

$$
\begin{aligned}
& \beta_{q}(t, x)=\int_{0}^{\frac{1-x}{\mu_{q}}} \sum_{p=q}^{n} \bar{\omega}_{q p}\left(\mu_{q} \nu+x\right) \beta^{p}\left(t-\nu, \mu_{q} \nu+x\right) \\
& +\sum_{p>r}^{n} \bar{\gamma}_{q p}\left(\mu_{q} \nu+x\right) h_{\left[0, a_{p q}\right]}\left(\mu_{q} \nu+x\right) \alpha^{p}\left(t-\nu, \mu_{q} \nu+x\right) d \nu
\end{aligned}
$$

with $1 \geq x>a_{r q}$, and $t \geq \frac{1-x}{\mu_{q}}$ (this explains why the last sum starts at $p>r$ ). Consequently, $\forall p \geq q$ and $\forall \nu \in\left[0, \frac{1-x}{\mu_{q}}\right]$ such that $\mu_{q} \nu+x \leq a_{p q}$ (in order to have $h_{\left[0, a_{p q}\right]}\left(\mu_{q} \nu+x\right) \neq 0$ ):

$$
\begin{aligned}
t \geq \frac{1-x}{\mu_{q}} & \Rightarrow t-\frac{x}{\lambda_{p}} \geq\left(\frac{\lambda_{p}}{\mu_{q}\left(\lambda_{p}+\mu_{q}\right)}-\frac{x}{\mu_{q}}\right)\left(1+\frac{\mu_{q}}{\lambda_{p}}\right) \\
& \Rightarrow t-\frac{x}{\lambda_{p}} \geq\left(\frac{a_{p q}}{\mu_{q}}-\frac{x}{\mu_{q}}\right)\left(1+\frac{\mu_{q}}{\lambda_{p}}\right) \\
& \Rightarrow t-\frac{x}{\lambda_{p}} \geq\left(1+\frac{\mu_{q}}{\lambda_{p}}\right) \nu \\
& \Rightarrow t-\nu \geq \frac{x+\mu_{q} \nu}{\lambda_{p}}
\end{aligned}
$$

Consequently, using the fact that $P(s-1)$ is true, it yields $\forall \nu \in\left[0, \frac{1-x}{\mu_{q}}\right]$ such that $\mu_{q} \nu+x \geq a_{p q}$

$$
\alpha^{p}\left(t-\nu, \mu_{q} \nu+x\right)=0
$$

Consequently the second sum in (36) is always null for $t \geq$ $\frac{1-x}{\mu_{q}}$. Moreover, using the fact that $P_{1}(q-1)$ is true, we can simplify the first sum removing most of the terms. We can rewrite (36) as:

$$
\beta^{q}(t, x)=\int_{0}^{\frac{1-x}{\mu_{q}}} \bar{\omega}_{q q}\left(\mu_{q} \nu+x\right) \beta^{q}\left(t-\nu, \mu_{q} \nu+x\right) d \nu
$$

with $1 \geq x>a_{r q}$, and $t \geq \frac{1-x}{\mu_{q}}$. Consequently $\beta^{q}(t, x)=0$ and $P_{1}(q)$ is true. This achieves the proof of $P_{1}(q)$ for all $1 \leq q \leq m$.

For a given $p$ we now focus on the following term of (34)

$$
\gamma_{r p}\left(x-\lambda_{r} \nu\right) h_{\left[a_{r p}, 1\right]}\left(x-\lambda_{r} \nu\right) \beta^{p}\left(t-\nu, x-\lambda_{r} \nu\right)
$$

This term can be non null only if

$$
x-\lambda_{r} \nu \geq a_{r p} \quad \forall \nu \in\left[0, \frac{x}{\lambda_{r}}\right]
$$

It yields

$$
\frac{\mu_{q}+\lambda_{r}}{\lambda_{r}} x-\left(\mu_{q}+\lambda_{r}\right) \nu \geq 1 \Leftrightarrow \frac{\mu_{q}}{\lambda_{r}} x+x-1 \geq\left(\mu_{q}+\lambda_{r}\right) \nu
$$

Since $t \geq \frac{x}{\lambda_{r}}$ it yields

$$
\mu_{q} t+x-1 \geq\left(\mu_{q}+\lambda_{r}\right) \nu \Rightarrow \mu_{q}(t-\nu) \geq 1-x+\lambda_{r} \nu
$$

Using $P_{1}$ we can deduce that (39) is always null. Consequently, combining this result with (35), we can rewrite (34) as

$$
\alpha^{s}(t, x)=\int_{0}^{\frac{x}{\lambda_{r}}} \omega_{r r}\left(x-\lambda_{r} \nu\right) \alpha^{r}\left(t-\nu, x-\lambda_{r} \nu\right)
$$

with $x \in[0,1]$ and $t \geq \frac{x}{\lambda_{r}}$. Consequently it yields

$$
\forall x \in[0,1], \quad t \geq \frac{x}{\lambda_{p}} \Rightarrow \alpha^{p}(x, t)=0
$$

It achieves the recursion. It is then quite straightforward to prove a similar result for $\beta$. Consequently we have

$$
\begin{aligned}
& \forall t \geq \frac{1}{\lambda_{1}}, \quad \alpha(x, t)=0 \\
& \forall t \geq \frac{1}{\mu_{1}}, \quad \beta(x, t)=0
\end{aligned}
$$


This concludes the proof.

Using an operator framework, system (19)-(20) rewrites as

$$
\frac{d}{d t}\left(\begin{array}{l}
\alpha \\
\beta
\end{array}\right)=A_{0}\left(\begin{array}{l}
\alpha \\
\beta
\end{array}\right)
$$

The operator $A_{0}$ is defined by

$$
\begin{aligned}
A_{0}: D\left(A_{0}\right) & \subset\left(L^{2}(0,1)\right)^{2} \rightarrow\left(L^{2}(0,1)\right)^{2} \\
& \left(\begin{array}{c}
\alpha \\
\beta
\end{array}\right) \longmapsto\left(\begin{array}{c}
-\Lambda^{+} \alpha_{x}+\Omega \alpha+\Gamma \beta \\
\Lambda^{-} \beta_{x}+\bar{\Omega} \beta+\bar{\Gamma} \alpha
\end{array}\right)
\end{aligned}
$$

with

$$
D\left(A_{0}\right)=\left\{(\alpha, \beta) \in\left(H^{1}(0,1)\right)^{2} \mid \alpha(0)=\beta(1)=0\right\}
$$

$A_{0}$ is well defined and its adjoint $A_{0}^{*}$ is

$$
\begin{aligned}
A_{0}^{*}: D\left(A_{0}^{*}\right) & \subset\left(L^{2}(0,1)\right)^{2} \rightarrow\left(L^{2}(0,1)\right)^{2} \\
& \left(\begin{array}{l}
\alpha \\
\beta
\end{array}\right) \longmapsto\left(\begin{array}{c}
\alpha_{x}^{T} \Lambda^{+}+\alpha^{T} \Omega+\beta^{T} \bar{\Gamma} \\
-\beta_{x}^{T} \Lambda^{-}+\beta^{T} \bar{\Omega}+\alpha^{T} \Gamma
\end{array}\right)^{T}
\end{aligned}
$$

with

$$
D\left(A_{0}^{*}\right)=\left\{(\alpha, \beta) \in\left(H^{1}(0,1)\right)^{2} \mid \alpha(1)=\beta(0)=0\right\}
$$

\section{B. Fredholm transformation}

\section{1) Definition of the transformation}

In order to map the original system (1)-(3) to the target system (19)-(21), we use the following transformation

$$
\begin{aligned}
& \alpha(t, x)=u(t, x) \\
& +h_{[0, \bar{a}]}(x) \int_{x}^{-\frac{\bar{\mu}}{\lambda} x+1}(K(x, \xi) u(t, \xi)+L(x, \xi) v(t, \xi)) d \xi \\
& +h_{] \bar{a}, 1]}(x) \int_{\frac{\bar{\lambda}}{\bar{\mu}}(1-x)}^{x}(M(x, \xi) u(t, \xi)+N(x, \xi) v(t, \xi)) d \xi \\
& \beta(t, x)=v(t, x) \\
& +h_{[0, \bar{a}]}(x) \int_{x}^{-\frac{\bar{\mu}}{\lambda} x+1}(\bar{K}(x, \xi) u(t, \xi)+\bar{L}(x, \xi) v(t, \xi)) d \xi \\
& +h_{] \bar{a}, 1]}(x) \int_{\frac{\bar{\lambda}}{\bar{\mu}}(1-x)}^{x}(\bar{M}(x, \xi) u(t, \xi)+\bar{N}(x, \xi) v(t, \xi)) d \xi
\end{aligned}
$$

where we recall that for any interval $\mathrm{I}, h_{I}(x)$ is defined by

$$
h_{I}(x)= \begin{cases}1 & \text { if } x \in I \\ 0 & \text { else }\end{cases}
$$

We define the following triangular domains, depicted in Figure 1 :

$$
\begin{array}{ll}
\mathcal{T}_{0}=\{(x, \xi) \mid & \left.x \in[0, \bar{a}], \quad x \leq \xi \leq-\frac{\bar{\mu}}{\bar{\lambda}} x+1\right\} \\
\overline{\mathcal{T}}_{1}=\{(x, \xi) \mid & \left.x \in[\bar{a}, 1], \quad \frac{\bar{\lambda}}{\bar{\mu}}(1-x)<\xi \leq x\right\}
\end{array}
$$

The kernels $K, L, \bar{K}$ and $\bar{L}$ are defined on $\mathcal{T}_{0}$. The kernels $M, N, \bar{M}$ and $\bar{N}$ are defined on $\overline{\mathcal{T}}_{1}$. They are assumed continuous in their domains of definition. They all have yet to be defined.
Remark 6: One may think that due to the use of the $h$ functions, the transformation presents a discontinuity in $x=\bar{a}$. Nevertheless, one can check that the right and left limits are equal since the integrals vanish and that consequently we do not have any discontinuity.

Remark 7: Since $\alpha(0)=\beta(1)=0$ the two control laws $U$ and $V$ can be computed as functions of $(u, v)$.

Remark 8: This transformation is a Fredholm transformation and can be rewritten using integrals between 0 and 1 as follows $\alpha(t, x)=u(t, x)-\int_{0}^{1} Q_{11}(x, \xi) u(t, \xi)+Q_{12}(x, \xi) v(t, \xi) d \xi$

$$
\beta(t, x)=v(t, x)-\int_{0}^{1} Q_{21}(x, \xi) u(t, \xi)+Q_{22}(x, \xi) v(t, \xi) d \xi
$$

with

$$
\begin{aligned}
Q_{11}(x, \xi) & =-K(x, \xi) h_{\left[x,-\frac{\bar{\mu}}{\lambda} x+1\right]}(\xi) h_{[0, \bar{a}]}(x) \\
& -M(x, \xi) h_{\left[\frac{\bar{\lambda}}{\bar{\mu}}(1-x), x\right]}(\xi) h_{] \bar{a}, 1]}(x) \\
Q_{12}(x, \xi) & =-L(x, \xi) h_{\left[x,-\frac{\bar{\mu}}{\lambda} x+1\right]}(\xi) h_{[0, \bar{a}]}(x) \\
& -N(x, \xi) h_{\left[\frac{\overline{\bar{\lambda}}}{\bar{\mu}}(1-x), x\right]}(\xi) h_{] \bar{a}, 1]}(x) \\
Q_{21}(x, \xi) & =-\bar{K}(x, \xi) h_{\left[x,-\frac{\bar{\mu}}{\lambda} x+1\right]}(\xi) h_{[0, \bar{a}]}(x) \\
& -\bar{M}(x, \xi) h_{\left[\frac{\bar{\lambda}}{\bar{\mu}}(1-x), x\right]}(\xi) h_{] \bar{a}, 1]}(x) \\
Q_{22}(x, \xi) & =-\bar{L}(x, \xi) h_{\left[x,-\frac{\bar{\mu}}{\lambda} x+1\right]}(\xi) h_{[0, \bar{a}]}(x) \\
& -\bar{N}(x, \xi) h_{\left[\frac{\bar{\lambda}}{\bar{\mu}}(1-x), x\right]}(\xi) h_{] \bar{a}, 1]}(x)
\end{aligned}
$$

2) Kernel equations

We now differentiate the Fredholm transformation (52)-(53) with respect to time and space to compute the equations satisfied by the kernels. We start with the $\alpha$-transformation (52).

If $x \leq \bar{a}$ : Differentiating (52) with respect to space and using the Leibniz rule yields

$$
\begin{aligned}
\alpha_{x}(t, x)= & u_{x}(t, x)-K(x, x) u(t, x)-L(x, x) v(t, x) \\
& -\frac{\bar{\mu}}{\bar{\lambda}} K\left(x,-\frac{\bar{\mu}}{\bar{\lambda}} x+1\right) u\left(-\frac{\bar{\mu}}{\bar{\lambda}} x+1\right) \\
& -\frac{\bar{\mu}}{\bar{\lambda}} L\left(x,-\frac{\bar{\mu}}{\bar{\lambda}} x+1\right) v\left(-\frac{\overline{\bar{\mu}}}{\bar{\lambda}} x+1\right) \\
+\int_{x}^{-\frac{\bar{\mu}}{\lambda} x+1} & \left(K_{x}(x, \xi) u(t, \xi)+L_{x}(x, \xi) v(t, \xi)\right) d \xi
\end{aligned}
$$

Differentiating (52) with respect to time, using (1), (2) and integrating by parts yields

$$
\begin{aligned}
& \alpha_{t}(t, x)=-\Lambda^{+} u_{x}(t, x)+\Sigma^{++} u(t, x)+\Sigma^{+-} v(t, x) \\
&+ K(x, x) \Lambda^{+} u(t, x)-L(x, x) \Lambda^{-} v(t, x) \\
&-K\left(x,-\frac{\bar{\mu}}{\bar{\lambda}} x+1\right) \Lambda^{+} u\left(t,-\frac{\bar{\mu}}{\bar{\lambda}} x+1\right) \\
&+L\left(x,-\frac{\overline{\bar{\mu}}}{\bar{\lambda}} x+1\right) \Lambda^{-} v\left(t,-\frac{\bar{\mu}}{\bar{\lambda}} x+1\right) \\
&+\int_{x}^{-\frac{\bar{\mu}}{\lambda} x+1}\left(K_{\xi}(x, \xi) \Lambda^{+} u(t, \xi)+L_{\xi}(x, \xi) \Lambda^{-} v(t, \xi)\right) d \xi
\end{aligned}
$$


Plugging these expressions into the target system (19)-(20) yields the following system of kernel equations

$$
\begin{aligned}
0= & \Sigma^{++}-\Lambda^{+} K(x, x)+K(x, x) \Lambda^{+}-\Omega(x) \\
0= & \Sigma^{+-}-\Lambda^{+} L(x, x)-L(x, x) \Lambda^{-}-\Gamma(x) \\
0= & -\frac{\bar{\mu}}{\bar{\lambda}} \Lambda^{+} L\left(x,-\frac{\bar{\mu}}{\bar{\lambda}} x+1\right)+L\left(x,-\frac{\bar{\mu}}{\bar{\lambda}} x+1\right) \Lambda^{-} \\
0= & \overline{\bar{\lambda}} \Lambda^{+} K\left(x,-\frac{\bar{\mu}}{\bar{\lambda}} x+1\right)+K\left(x,-\frac{\bar{\mu}}{\bar{\lambda}} x+1\right) \Lambda^{+} \\
0= & \Lambda^{+} K_{x}(x, \xi)+K_{\xi}(x, \xi) \Lambda^{+}+K(x, \xi) \Sigma^{++} \\
& +L(x, \xi) \Sigma^{-+}-\Omega(x) K(x, \xi)-\Gamma(x) \bar{K}(x, \xi) \\
0= & \Lambda^{+} L_{x}(x, \xi)-L_{\xi}(x, \xi) \Lambda^{-}+K(x, \xi) \Sigma^{+-} \\
& +L(x, \xi) \Sigma^{--}-\Omega(x) L(x, \xi)-\Gamma(x) \bar{L}(x, \xi) \\
&
\end{aligned}
$$

if $x>\bar{a}$ : Similarly we get

$$
\begin{aligned}
0= & \Sigma^{++}+\Lambda^{+} M(x, x)-M(x, x) \Lambda^{+}-\Omega(x) \\
0= & \Sigma^{+-}+\Lambda^{+} N(x, x)+N(x, x) \Lambda^{-}-\Gamma(x) \\
0= & -\frac{\bar{\lambda}}{\bar{\mu}} \Lambda^{+} N\left(x,-\frac{\bar{\lambda}}{\bar{\mu}}(x-1)\right)+N\left(x,-\frac{\bar{\lambda}}{\bar{\mu}}(x-1)\right) \Lambda^{-} \\
0= & \frac{\bar{\lambda}}{\bar{\mu}} \Lambda^{+} M\left(x,-\frac{\bar{\lambda}}{\bar{\mu}}(x-1)\right)+M\left(x,-\frac{\bar{\lambda}}{\bar{\mu}}(x-1)\right) \Lambda^{+} \\
0= & \Lambda^{+} M_{x}(x, \xi)+M_{\xi}(x, \xi) \Lambda^{+}+M(x, \xi) \Sigma^{++} \\
& +N(x, \xi) \Sigma^{-+}-\Omega(x) M(x, \xi)-\Gamma(x) \bar{M}(x, \xi) \\
0= & \Lambda^{+} N_{x}(x, \xi)-N_{\xi}(x, \xi) \Lambda^{-}+M(x, \xi) \Sigma^{+-} \\
& +N(x, \xi) \Sigma^{--}-\Omega(x) N(x, \xi)-\Gamma(x) \bar{N}(x, \xi)
\end{aligned}
$$

We now compute the kernels for the $\beta$-transformation if $x \leq \bar{a}$ : Differentiating (53) with respect to space and time and then plugging into the target system (19)-(20) yields the following system of kernel equations

$$
\begin{aligned}
0= & \Sigma^{-+}+\Lambda^{-} \bar{K}(x, x)+\bar{K}(x, x) \Lambda^{+}-\bar{\Gamma}(x) \\
0= & \Sigma^{--}-\Lambda^{-} \bar{L}(x, x)+\bar{L}(x, x) \Lambda^{-}-\bar{\Omega}(x) \\
0= & -\bar{K}\left(x,-\frac{\bar{\mu}}{\lambda_{m}} x+1\right) \Lambda^{+}+\frac{\bar{\mu}}{\bar{\lambda}} \Lambda^{-} \bar{K}\left(x,-\frac{\bar{\mu}}{\bar{\lambda}} x+1\right) \\
0= & \overline{\bar{\mu}} \Lambda^{-} \bar{L}\left(x,-\frac{\bar{\mu}}{\bar{\lambda}} x+1\right)+\bar{L}\left(x,-\frac{\bar{\mu}}{\lambda_{m}} x+1\right) \Lambda^{-} \\
0= & -\Lambda^{-} \bar{K}_{x}(x, \xi)+\bar{K}_{\xi}(x, \xi) \Lambda^{+}+\bar{K}(x, \xi) \Sigma^{++} \\
& +\bar{L}(x, \xi) \Sigma^{-+}-\bar{\Omega}(x) \bar{K}(x, \xi)-\bar{\Gamma}(x) K(x, \xi) \\
0= & -\Lambda^{-} \bar{L}_{x}(x, \xi)-\bar{L}_{\xi}(x, \xi) \Lambda^{-}+\bar{K}(x, \xi) \Sigma^{+-} \\
& +\bar{L}(x, \xi) \Sigma^{--}-\bar{\Omega}(x) \bar{L}(x, \xi)-\bar{\Gamma}(x) L(x, \xi) \\
&
\end{aligned}
$$

if $x>\bar{a}$ : Similarly, we get

$$
\begin{aligned}
& 0=\Sigma^{-+}-\Lambda^{-} \bar{M}(x, x)-\bar{M}(x, x) \Lambda^{+}-\bar{\Gamma}(x) \\
& 0=\Sigma^{--}+\Lambda^{-} \bar{N}(x, x)-\bar{N}(x, x) \Lambda^{-}-\bar{\Omega}(x) \\
& 0=\frac{\bar{\lambda}}{\bar{\mu}} \Lambda^{-} \bar{N}\left(x,-\frac{\bar{\lambda}}{\bar{\mu}}(x-1)\right)+\bar{N}\left(x,-\frac{\bar{\lambda}}{\bar{\mu}}(x-1)\right) \Lambda^{-}
\end{aligned}
$$

$$
\begin{aligned}
0= & -\frac{\bar{\lambda}}{\bar{\mu}} \Lambda^{-} \bar{M}\left(x,-\frac{\bar{\lambda}}{\bar{\mu}}(x-1)\right)+\bar{M}\left(x,-\frac{\bar{\lambda}}{\bar{\mu}}(x-1)\right) \Lambda^{+} \\
0= & -\Lambda^{-} \bar{M}_{x}(x, \xi)+\bar{M}_{\xi}(x, \xi) \Lambda^{+}+\bar{M}(x, \xi) \Sigma^{++} \\
& +\bar{N}(x, \xi) \Sigma^{-+}-\bar{\Omega}(x) \bar{M}(x, \xi)-\bar{\Gamma}(x) M(x, \xi) \\
0= & -\Lambda^{-} \bar{N}_{x}(x, \xi)-\Lambda^{-} \bar{N}_{\xi}(x, \xi)+\bar{M}(x, \xi) \Sigma^{+-} \\
& +\bar{N}(x, \xi) \Sigma^{--}-\bar{\Omega}(x) \bar{N}(x, \xi)-\bar{\Gamma}(x) N(x, \xi)
\end{aligned}
$$

The well-posedness of all these kernel equations is assessed in the following theorems

Theorem 1: Consider system (65)-(70) and (77)-(82). There exists a unique solution $K, L, \bar{K}$ and $\bar{L}$ in $L^{\infty}\left(\mathcal{T}_{0}\right)$

Theorem 2: Consider system (71)-(76) and (83)-(88). There exists a unique solution $M, N, \bar{M}$ and $\bar{N}$ in $L^{\infty}\left(\mathcal{T}_{1}\right)$

The proof of these theorems is described in the following section and uses the cascade structure of the kernel equations (which is due to the particular shapes of the matrices $\Omega, \bar{\Omega}, \Gamma$ and $\bar{\Gamma}$ )

\section{WELL-POSEDNESS OF THE KERNEL EQUATIONS}

In this section we prove Theorem 1. The proof of Theorem 2 is quite similar and is not detailed here. The proof follows three steps:

- First, we develop the kernel equations and the associated boundary conditions.

- Then we define a particular sequence in which to solve the equations using the properties of the matrices $\Gamma$ and $\bar{\Gamma}$.

- Finally, this sequence is used in a recursive approach to complete the proof.

For every iteration of the recursion we prove that the $i^{\text {th }}$ line of the system of kernel PDEs $K_{i j}, L_{i j}$ (resp. $\bar{K}_{i j}, \bar{L}_{i j}$ ) is well-posed. This proof involves the following well-posedness theorem.

Theorem 3: Consider the following system of hyperbolic equations

$$
\epsilon_{j} \partial_{x} F_{j}(x, \xi)+\nu_{j} \partial_{\xi} F_{j}(x, \xi)=\Sigma_{j}(x, \xi) F(x, \xi)
$$

where $F=\left(F_{1} \ldots F_{n+m}\right)$ is defined on the triangle $\mathcal{D}$ :

$$
\mathcal{D}=\left\{(x, \xi) \mid x \leq \xi \quad c_{1} \xi \leq c_{1}-c_{2} x \quad d_{1} \xi \geq d_{1}-d_{2} x\right\}
$$

where the coefficients $c_{1}, c_{2}, d_{1}, d_{2}$ are all positive. The corresponding boundary conditions are defined on a closed subset $\mathcal{R}_{j}$ included on the boundary of the domain $\partial \mathcal{D}$

$$
F_{j \mid \mathcal{R}_{j}}=f_{j}
$$

Assume

- that the homogeneous system, obtained by taking $\Sigma(x, \xi)=0$ in (89) along with boundary conditions (91) is well-posed;

- that there exists $\alpha_{j}>0$ such that the following inequalities holds for all $j=1, \ldots, n+m$

$$
\forall(x, \xi) \in \mathcal{D} \quad \alpha_{j} \epsilon_{j}(x)+\nu_{j}(\xi)<-\delta<0
$$

Then the system (89) with boundary conditions (91) has an unique solution $F \in L^{\infty}(\mathcal{D})$. 
Proof: The proof of this theorem is given in Appendix.

Remark 9: A necessary and sufficient condition for the first assumption to be satisfied is that, for every $j=1 \ldots n+m$ the characteristics defined by the $\epsilon_{j}, \nu_{j}$ uniquely connect each point of $\mathcal{T}_{0}$ to $\mathcal{D}_{j}$.

\section{A. Development of the kernel equations}

We only focus on the kernels $K, L, \bar{K}$ and $\bar{L}$ defined on $\mathcal{T}_{0}$ since the proof is similar for the remaining kernels. Developing (65)-(70) and (77)-(82) we get the following set of kernel PDEs:

$$
\text { for } 1 \leq i \leq n, 1 \leq j \leq n
$$

$$
\begin{gathered}
\lambda_{i} \partial_{x} K_{i j}(x, \xi)+\lambda_{j} \partial_{\xi} K_{i j}(x, \xi)=-\sum_{k=1}^{n} \sigma_{k j}^{++} K_{i k}(x, \xi) \\
-\sum_{p=1}^{m} \sigma_{p j}^{-+} L_{i p}(x, \xi)+\sum_{i \leq p \leq n} K_{p j}(x, \xi) \omega_{i p}(x) \\
+\sum_{1 \leq p \leq m} \bar{K}_{p j}(x, \xi) \Gamma_{i p}(x)
\end{gathered}
$$

for $1 \leq i \leq n, 1 \leq j \leq m$

$$
\begin{array}{r}
\lambda_{i} \partial_{x} L_{i j}(x, \xi)-\mu_{j} \partial_{\xi} L_{i j}(x, \xi)=-\sum_{k=1}^{m} \sigma_{k j}^{--} L_{i k}(x, \xi) \\
-\sum_{p=1}^{n} \sigma_{p j}^{+-} K_{i p}(x, \xi)+\sum_{i \leq p \leq n} L_{p j}(x, \xi) \omega_{i p}(x) \\
+\sum_{1 \leq p \leq m} \bar{L}_{p j}(x, \xi) \Gamma_{i p}(x)
\end{array}
$$

for $1 \leq i \leq m, 1 \leq j \leq n$

$$
\begin{array}{r}
\mu_{i} \partial_{x} \bar{K}_{i j}(x, \xi)-\lambda_{j} \partial_{\xi} \bar{K}_{i j}(x, \xi)=\sum_{k=1}^{n} \sigma_{k j}^{++} \bar{K}_{i k}(x, \xi) \\
+\sum_{p=1}^{m} \sigma_{p j}^{-+} \bar{L}_{i p}(x, \xi)-\sum_{i \leq p \leq m} \bar{K}_{p j}(x, \xi) \bar{\omega}_{i p}(x) \\
-\sum_{1 \leq p \leq n} K_{p j}(x, \xi) \bar{\Gamma}_{i p}(x)
\end{array}
$$

for $1 \leq i \leq m, 1 \leq j \leq m$

$$
\begin{array}{r}
\mu_{i} \partial_{x} \bar{L}_{i j}(x, \xi)+\mu_{j} \partial_{\xi} \bar{L}_{i j}(x, \xi)=\sum_{k=1}^{m} \sigma_{k j}^{--} \bar{L}_{i k}(x, \xi) \\
+\sum_{p=1}^{n} \sigma_{p j}^{+-} \bar{L}_{i p}(x, \xi)-\sum_{i \leq p \leq m} \bar{L}_{p j}(x, \xi) \bar{\omega}_{i p}(x) \\
-\sum_{1 \leq p \leq n} L_{p j}(x, \xi) \bar{\Gamma}_{i p}(x)
\end{array}
$$

with the following set of boundary conditions (in order to make the whole content more readable we have removed the domains of definition of the indices)

$$
\begin{aligned}
& K_{i j}\left(x, 1-\frac{\bar{\mu}}{\bar{\lambda}} x\right)=0 \\
& K_{i j}(x, x)=\frac{\sigma^{++}}{\lambda_{i}-\lambda_{j}} \quad i>j \\
& \bar{L}_{i j}\left(x,-\frac{\bar{\mu}}{\bar{\lambda}} x+1\right)=0 \\
& \bar{L}_{i j}(x, x)=\frac{\sigma^{--}}{\mu_{i}-\mu_{j}} \quad i>j \\
& \left(\frac{\mu_{j}}{\lambda_{i}}-\frac{\bar{\mu}}{\bar{\lambda}}\right) L_{i j}\left(x, 1-\frac{\bar{\mu}}{\bar{\lambda}} x\right)=0 \\
& \left(-\lambda_{i}+\mu_{i} \frac{\bar{\mu}}{\bar{\lambda}}\right) \bar{K}_{i j}\left(x,-\frac{\bar{\mu}}{\lambda_{m}} x+1\right)=0 \\
& \forall x<a_{i j} \quad L_{i j}(x, x)=-\frac{\sigma_{i j}^{+-}}{\lambda_{i}+\mu_{j}} \\
& \text { if } 1-a_{j i} \geq \bar{a} \quad \bar{K}_{i j}(x, x)=-\frac{\sigma^{-+}}{\lambda_{j}+\mu_{i}}
\end{aligned}
$$

We add the following arbitrary boundary conditions (in order to have a well-posed system)

$$
\text { if } 1-a_{j i}<\bar{a} \quad \bar{K}_{i j}(0, \xi)=0
$$

Besides, (65) imposes

$$
\forall i \leq j \quad \omega_{i j}(x)=\left(\lambda_{j}-\lambda_{i}\right) K_{i j}(x, x)+\sigma_{i j}^{++}
$$

and (66) imposes

$$
\forall a_{i j}<x<1 \quad \gamma_{i j}(x)=-\left(\lambda_{i}+\mu_{j}\right) L_{i j}(x, x)+\sigma_{i j}^{+-}
$$

Similarly (78) imposes

$$
\forall i \leq j \quad \bar{\omega}_{i j}(x)=\left(\mu_{j}-\mu_{i}\right) \bar{L}_{i j}(x, x)+\sigma_{i j}^{--}
$$

and (77) imposes

$$
\forall 0 \leq x \leq a_{j i} \quad \bar{\gamma}_{i j}(x)=\left(\mu_{i}+\lambda_{j}\right) \bar{K}_{i j}(x, x)+\sigma_{i j}^{-+}
$$

This induces a coupling between the kernels through equations (93), (94), (95) and (96) that could appear as non linear at first sight. However, the coupling has a linear cascade structure due to the particular shapes of the matrices $\Omega, \bar{\Omega}, \Gamma$ and $\bar{\Gamma}$. We now define two sequences $r_{i}$ and $\bar{r}_{i}$ that will be used in a recursive proof of the well-posedness. Some of these equations with the corresponding characterisitc lines are represented on Figure 1 and Figure 2.

Remark 10: The artificial boundary condition we add for the kernel $\bar{K}$ is not a degree of freedom since it has no impact on the control law and on the stability of the target system. 


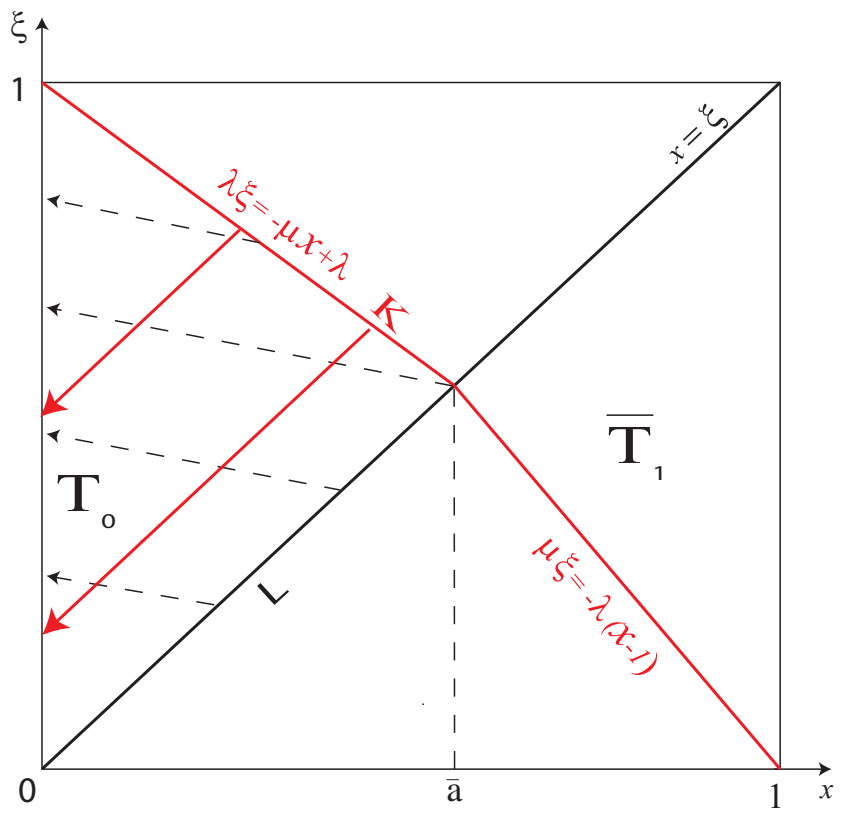

Fig. 1. Representation of the K-kernels and the L-kernels $\left(a_{i j} \geq \bar{a}\right)$

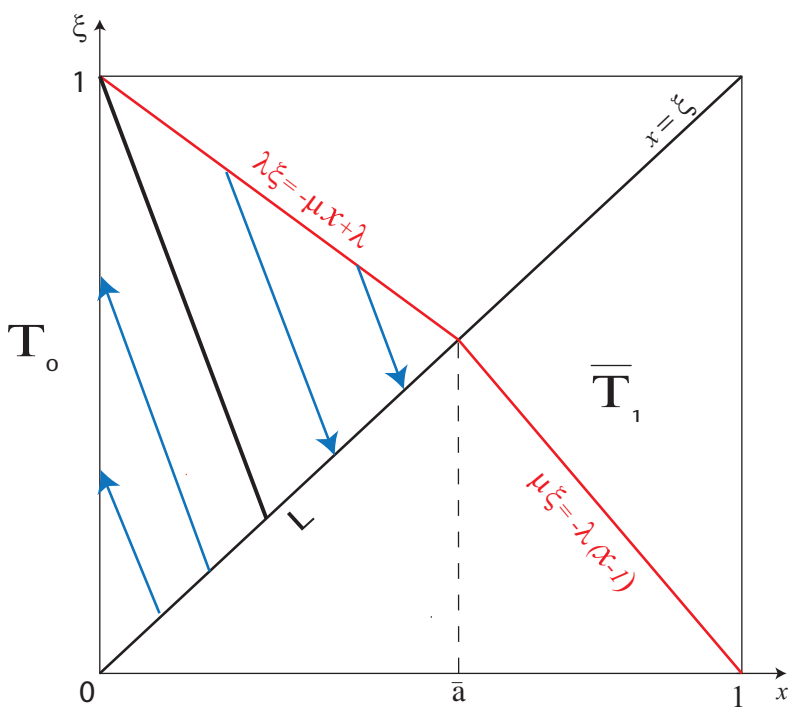

Fig. 2. Representation of the L-kernels $\left(a_{i j}<\bar{a}\right)$

\section{B. Definition of the sequences $r_{i}$ and $\bar{r}_{i}$}

In this subsection we define two sequences $r_{i}$ and $\bar{r}_{i}$ that we are going to use in the recursive proof. Let us consider the matrices $\Delta$ and $\bar{\Delta}$ defined by the following relations

$$
\begin{gathered}
\forall 1 \leq i \leq n \forall 1 \leq j \leq m \Delta_{i j}= \begin{cases}0 & \text { if } a_{i j} \geq \bar{a} \\
1 & \text { else }\end{cases} \\
\forall 1 \leq i \leq m \forall 1 \leq j \leq n \bar{\Delta}_{i j}= \begin{cases}0 & \text { if } a_{j i}<1-\bar{a} \\
1 & \text { else }\end{cases}
\end{gathered}
$$

These matrices have exactly the same structure as the matrices $\Gamma$ and $\bar{\Gamma}$. We have the following results (some of the proofs are quite straightforward and are omitted there).

Lemma 2: $\bar{\Delta}=1-\Delta^{T}$

Proof: The proof relies on the fact that due to the definition of $\bar{a}$ (Equation (10)), if $a_{i j}>\bar{a}$ then $a_{i j}>1-\bar{a}$. Suppose that $\Delta_{i j}=0$, then $a_{i j} \geq \bar{a} \Rightarrow a_{i j} \geq 1-\bar{a}$ (since $\bar{a}>\frac{1}{2}$ ). It yields $\bar{\Delta}_{j i}=1$.

Suppose now that $\Delta_{i j}=1$, then $a_{i j}<\bar{a} \Rightarrow a_{i j}<1-\bar{a}$ (due to the definition of $\bar{a}$ ). It yields $\bar{\Delta}_{j i}=0$.

Lemma 3: If $\Delta_{i j}=0$ then $\forall k>i \quad \Delta_{k j}=0$

Lemma 4: If $\Delta_{i j}=0$ then $\forall k<j \quad \Delta_{i k}=0$

The two previous lemmas use the fact that $a_{i j}<a_{i+1, j}$ and $a_{i j}>a_{i, j+1}$. Same results hold for $\bar{\Delta}$.

Lemma 5: Either the last line of $\Delta$ or the last line of $\bar{\Delta}$ is null.

Proof: Let us assume that the last line of $\bar{\Delta}$ is non-null. Consequently, $\forall j \in[1, n] a_{j m} \leq 1-\bar{a}$ and particularly $a_{n m} \leq$ $1-\bar{a}$. This implies $a_{n m} \leq \bar{a}$ (due to the definition of $\bar{a}$ ) and it yields $\Delta_{m n}=0$. Using the previous Lemma concludes the proof.

In the following we denote $s_{i}$ (resp $\bar{s}_{i}$ ) the number of coefficients which are equal to 1 in the $i^{\text {th }}$ line of $\Delta$ (resp $\bar{\Delta})$. For $0 \leq i \leq n+m$, we define the sequences $r_{i}$ and $\bar{r}_{i}$

$$
\left\{\begin{array}{lll}
\text { if } \bar{r}_{0}-\bar{r}_{i} \geq s_{r_{i}-1} & \text { then } \quad r_{i+1}=r_{i}-1 & \bar{r}_{i+1}=\bar{r}_{i} \\
\text { if } r_{0}-r_{i} \geq \bar{s}_{\bar{r}_{i}-1} & \text { then } \quad \bar{r}_{i+1}=\bar{r}_{i}-1 & r_{i+1}=r_{i}
\end{array}\right.
$$

where $r_{0}=n+1, \bar{r}_{0}=m+1$. We use the convention $s_{0}=$ $\bar{s}_{0}=\infty$.

Theorem 4: The sequences $r_{i}, \bar{r}_{i}$ are well defined. Moreover $r_{n+m}=\bar{r}_{n+m}=1$

Proof: To prove that the sequences are well defined we need to prove that for any $0 \leq i \leq n+m$ one and one only of the following assumptions is true

$$
\begin{aligned}
& \bar{r}_{0}-\bar{r}_{i} \geq s_{r_{i}-1} \\
& r_{0}-r_{i} \geq \bar{s}_{\bar{r}_{i}-1}
\end{aligned}
$$

We start by proving that at least one of the two assumptions is true. By contradiction let us assume that none of them hold. Consequently we have, for some $\mathrm{i}$

$$
\begin{aligned}
& \bar{r}_{0}-\bar{r}_{i}<s_{r_{i}-1} \\
& r_{0}-r_{i}<\bar{s}_{\bar{r}_{i}-1}
\end{aligned}
$$

- By definition of $s_{r_{i}-1}$, we have exactly $s_{r_{i}-1}$ coefficients that are equal to 1 on the $\left(r_{i}-1\right)^{t h}$ line of the matrix $\Delta$. Using Lemma 2 yields that we have exactly $m-s_{r_{i}-1}$ coefficients equal to 1 on the $\left(r_{i}-1\right)^{t h}$ column of $\bar{\Delta}$

- By definition of $\bar{s}_{\bar{r}_{i}-1}$, we have exactly $\bar{s}_{\bar{r}_{i}-1}$ coefficients that are equal to 1 on the $\left(\bar{r}_{i}-1\right)^{\text {th }}$ line of $\bar{\Delta}$. Consequently $\bar{\Delta}_{\bar{r}_{i}-1, \bar{s}_{\bar{r}_{i}-1}}=1$. Adjusting Lemma 3 to $\bar{\Delta}$ yields that on the $\left(n+1-\bar{s}_{\bar{r}_{i}-1}\right)^{t h}$ column of $\bar{\Delta}$ we have at least $\bar{r}_{i}-1$ coefficients equal to 1 .

- Since $n+1-\bar{s}_{\bar{r}_{i}}<r_{i}$ we get $n+1-\bar{s}_{\bar{r}_{i}} \leq r_{i}-1$. It means that the column $n+1-\bar{s}_{\bar{r}_{i}}$ is located lefter than the column $r_{i}-1$. Consequently, using Lemma 3 , we must have a larger 
number of coefficients equal to 1 on the column $r_{i}-1$ than on the column $n+1-\bar{s}_{\bar{r}_{i}}$. This implies

$$
m-s_{r_{i}-1} \geq \bar{r}_{i}-1
$$

which is a contradiction with (114). To achieve the proof of the well posedness of the two sequences $r_{i}$ and $\bar{r}_{i}$, we need to prove that the two assumptions (112)-(113) cannot both be true. This is quite straightforward using the same ideas. If we assume that (113) holds, then $n+1-\bar{s}_{\bar{r}_{i}} \geq r_{i}$ implies that the column $n+1-\bar{s}_{\bar{r}_{i}}$ of $\bar{\Delta}$ is located strictly righter than the column $r_{i}-1$ and that consequently (Lemma 4 ), the number of coefficients equal to 1 on the former column is larger than the number on the later. This implies

$$
m-s_{r_{i}-1}<\bar{r}_{i}-1
$$

and consequently (112) is false. The same holds when (112) is true.

The following lemma makes the link between the matrices $\Delta, \bar{\Delta}$, and $\Gamma, \bar{\Gamma}$.

Lemma 6: The matrix $\Gamma(x)$ has at least $m-s_{i}$ nullcoefficients on its $i^{\text {th }}$ line. Similarly, the matrix $\bar{\Gamma}(x)$ has at least $n-\bar{s}_{i}$ null-coefficients on its $i^{\text {th }}$ line.

Corollary 1: $\forall i \leq n, \forall j \leq m-s_{i}, \Gamma(x)_{i j}=0$ and $\forall i \leq$ $m, \forall j \leq n-\bar{s}_{i}, \bar{\Gamma}(x)_{i j}=0$

Proof: The proofs of this lemma and of this corollary are quite straightforward noticing that the matrices $\Gamma$ (resp. $\bar{\Gamma})$ and $\Delta$ (resp. $\bar{\Delta}$ ) have exactly the same structure and that consequently the properties described above can be easily extended to $\Gamma$ and $\bar{\Gamma}$.

\section{Induction hypothesis}

By induction, let us consider the following property $P(q)$ defined for all $1 \leq q \leq m+n$ :

$\forall r_{q} \leq i \leq n, \forall \bar{r}_{q} \leq \bar{i} \leq m, \forall 1 \leq j \leq n, \forall 1 \leq d \leq m$, $\forall 1 \leq \bar{j} \leq n, \forall 1 \leq \bar{d} \leq m$, the problem (93)-(104) where $\Omega$, $\bar{\Omega}, \Gamma$ and $\bar{\Gamma}$ are defined by (106)-(108) has an unique solution $K_{i j}(\cdot, \cdot), L_{i d}(\cdot, \cdot), \bar{K}_{\bar{i} \bar{j}}(\cdot, \cdot), \bar{L}_{\bar{i} \bar{d}}(\cdot, \cdot) \in L^{\infty}\left(\mathcal{T}_{0}\right)$.

Initialization: For $q=1$, we have either $\left(r_{1}=n\right.$ and $\left.\bar{r}_{1}=m+1\right)$ or $\left(r_{1}=n+1\right.$ and $\left.\bar{r}_{1}=m\right)$ due to Theorem 3 . Assuming that $r_{1}=n$ and $\bar{r}_{1}=m+1$, system (93)-(104) rewrites as follow

for $1 \leq j \leq n$

$$
\begin{gathered}
\lambda_{n} \partial_{x} K_{n j}(x, \xi)+\lambda_{j} \partial_{\xi} K_{n j}(x, \xi)=-\sum_{k=1}^{n} \sigma_{k j}^{++} K_{n k}(x, \xi) \\
-\sum_{p=1}^{m} \sigma_{p j}^{-+} L_{n p}(x, \xi)+K_{n j}(x, \xi) \sigma_{n n}^{++}
\end{gathered}
$$

\section{for $1 \leq j \leq m$}

$$
\begin{gathered}
\lambda_{n} \partial_{x} L_{n j}(x, \xi)-\mu_{j} \partial_{\xi} L_{n j}(x, \xi)=-\sum_{k=1}^{m} \sigma_{k j}^{--} L_{n k}(x, \xi) \\
-\sum_{p=1}^{n} \sigma_{p j}^{+-} K_{n p}(x, \xi)+L_{n j}(x, \xi) \sigma_{n n}^{++}
\end{gathered}
$$

with the corresponding set of boundary conditions. The wellposedness of such system is quite straightforward using [18] or Theorem 3. The initialization still holds for $r_{1}=n+1$ and $\bar{r}_{1}=m$.

Induction: Let us assume that the property $P(q-1)$ $(1<q \leq n+m-1)$ is true. We consequently have that $\forall r_{q-1} \leq i \leq n, \forall \bar{r}_{q-1} \leq \bar{i} \leq n, \forall 1 \leq j \leq n, \forall 1 \leq d \leq m$, $\forall 1 \leq \bar{j} \leq n, \forall 1 \leq \bar{d} \leq m, K_{i j}(\cdot, \cdot), L_{i d}(\cdot, \cdot), \bar{K}_{\bar{i} \bar{j}}(\cdot, \cdot)$, and $\bar{L}_{\bar{i} \bar{d}}(\cdot, \cdot)$ are bounded.

In the following we assume that $\bar{r}_{q}=\bar{r}_{q-1}$ (and that consequently $r_{q}=r_{q-1}-1$ ). The result still holds if $r_{q}=r_{q-1}$ and the proof is similar. We denote $i=r_{q}$.

Using the induction hypothesis yields that $\forall 1 \leq \bar{j} \leq n$, $\forall 1 \leq \bar{d} \leq m, \forall \bar{r}_{q}=\bar{r}_{q-1} \leq \bar{i} \leq n \bar{K}_{\bar{i} \bar{j}}(\cdot, \cdot)$, and $\overline{\bar{L}}_{\bar{i} \bar{d}}(\cdot, \cdot)$ are well-posed.

Rewriting equation (93) yields

$$
\begin{gathered}
-\lambda_{i} \partial_{x} K_{i j}(x, \xi)-\lambda_{j} \partial_{\xi} K_{i j}(x, \xi)=\sum_{k=1}^{n} \sigma_{k j}^{++} K_{i k}(x, \xi) \\
+\sum_{p=1}^{m} \sigma_{p j}^{-+} L_{i p}(x, \xi)-\sum_{i \leq p \leq m} K_{p j}(x, \xi) \\
\left.\cdot\left(\left(\lambda_{p}-\lambda_{i}\right) K_{i p}(x, x)+\sigma_{i p}^{++}\right)\right] \\
-\sum_{1 \leq p \leq m} \bar{K}_{p j}(x, \xi) h_{\left[a_{i p}, 1\right]}(x) \cdot\left(\left(\lambda_{i}+\mu_{p}\right) L_{i p}(x, x)+\sigma_{i p}^{+-}\right)
\end{gathered}
$$

with the boundary conditions

$$
K_{i j}\left(x, 1-\frac{\bar{\mu}}{\bar{\lambda}} x\right)=0 \quad K_{i j}(x, x)=\frac{\sigma^{++}}{\lambda_{i}-\lambda_{j}} \quad i>j
$$

The one-but-last sum uses the expression of $K_{p j}$ for $i \leq$ $p \leq m$. This term is known and bounded for $p>i$ (induction assumption). For $p=i, \lambda_{i}=\lambda_{p}$ and the term $\left(\lambda_{p}-\lambda_{i}\right) K_{i p}\left(x_{i j}(x, \xi, s), x_{i j}(x, \xi, s)\right)$ cancels.

Using Corollary 1 and relation (107) it is possible to rewrite the last sum as

$$
\sum_{m+1-s_{i} \leq p \leq m} \bar{K}_{p j}(x, \xi)\left(\sigma_{i p}^{+-}+\left(\lambda_{i}+\mu_{p}\right) L_{i p}(x, \xi)\right) h_{\left[a_{i j}, 1\right]}(x)
$$

Using the definition of $r_{i}$, we have

$$
\begin{gathered}
s_{i} \leq s_{i-1}=s_{r_{q}-1} \leq m+1-\bar{r}_{q} \\
\quad \Rightarrow m-s_{i} \geq \bar{r}_{q}-1
\end{gathered}
$$

Consequently, the last sum uses the expression of $\bar{K}_{p j}$ for $\bar{r}_{q} \leq$ $p \leq m$ which is known according to the induction assumption. Therefore, all the non-linearites that could appear at first sight 
on the kernel equations actually involve terms that have been computed in the previous iterations and that are bounded. We can rewrite (120) as

$$
\begin{aligned}
-\lambda_{i} \partial_{x} K_{i j}(x, \xi) & -\lambda_{j} \partial_{\xi} K_{i j}(x, \xi)=\sum_{k=1}^{n} C_{k j}^{++}(x, \xi) K_{i k}(x, \xi) \\
& +\sum_{k=1}^{m} C_{k j}^{-+}(x, \xi) L_{i k}(x, \xi) d s
\end{aligned}
$$

where the coefficients $C_{k j}^{++}$and $C_{k j}^{-+}$are known and bounded (since they are either constants or computed during the previous iteration of the recursion).

Similarly, we can rewrite (94) as

$$
\begin{aligned}
-\lambda_{i} \partial_{x} L_{i j}(x, \xi) & +\mu_{j} \partial_{\xi} L_{i j}(x, \xi)=\sum_{k=1}^{n} C_{k j}^{-+}(x, \xi) K_{i k}(x, \xi) \\
& +\sum_{k=1}^{m} C_{k j}^{--}(x, \xi) L_{i k}(x, \xi) d s
\end{aligned}
$$

where the coefficients $C_{k j}^{-+}$and $C_{k j}^{--}$are known and bounded. Moreover we have the boundary condition

$$
\left(\frac{\mu_{j}}{\lambda_{i}}-\frac{\bar{\mu}}{\bar{\lambda}}\right) L_{i j}\left(x, 1-\frac{\bar{\mu}}{\bar{\lambda}} x\right)=0
$$

and

$$
\forall x<a_{i j}=\frac{\lambda_{i}}{\lambda_{i}+\mu_{j}} \quad L_{i j}(x, x)=\frac{\sigma_{i j}^{+-}}{\lambda_{i}+\mu_{j}}
$$

Each $L_{i j}$ has a discontinuity line defined by $\xi=1-\frac{\mu_{j}}{\lambda_{i}} x$. The characteristics are integrated in opposite directions on each side of the discontinuity: away from the $\xi=x$ boundary for $\xi \leq 1-\frac{\mu_{j}}{\lambda_{i}} x$ and away from the line $\xi=1-\frac{\bar{\mu}}{\lambda}$ for $\xi \geq 1-\frac{\mu_{j}}{\lambda_{i}} x$. Therefore the parameters $\alpha$ and $\delta$ of Theorem 3, which have to satisfy (92) for all $L_{i j}$ on the domain on which the equations are considered, vary on each side of the discontinuity of all the kernels. Therefore, in what follows, we define a sequence of triangular domains, depicted on Figure 3, on which there exists $\alpha$ and $\delta$ satisfying (92). More precisely, assuming that $a_{i 1} \geq \bar{a}$ (this specific case will be presented in Remark 10), for all $k \leq m+1$ such that $a_{i k}<\bar{a}$ (with the convention $\left.a_{i(m+1)}=0\right)$, consider the domains $\mathcal{T}_{k}$ :

$$
\begin{aligned}
\mathcal{T}_{k} & =\left\{(x, \xi) \mid x \leq \xi \quad \xi \leq 1-\frac{\mu_{k-1}}{\lambda_{i}} x \quad \xi \geq 1-\frac{\mu_{k}}{\lambda_{i}} x\right\} \\
\mathcal{T}_{m+1} & =\left\{(x, \xi) \mid 0 \leq x \leq \xi \quad \xi \leq 1-\frac{\mu_{m}}{\lambda_{i}} x\right\}
\end{aligned}
$$

The equations can be solved successively on these triangles, starting from the rightmost one. Their are represented on Figure 3.

The trace of the solution on the boundary of a given $\mathcal{T}_{k}$ provides boundary conditions of the system considered on $\mathcal{T}_{k+1}$.

By induction, let us now consider the property $\mathcal{Q}(k)$ defined for all $k$ such that $a_{i k}<\bar{a}$ by: The system (123)-(126) is well-posed on $\mathcal{T}_{k} \cap \mathcal{T}_{0}$.

Initialization: Let $k_{0}$ such that $a_{i k_{0}}<\bar{a} \leq a_{i\left(k_{0}-1\right)}$.

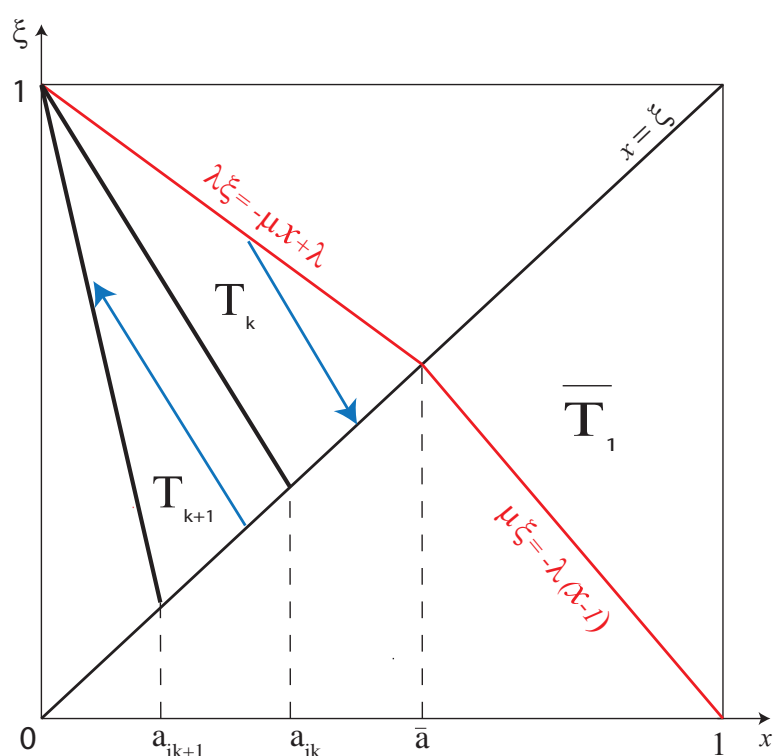

Fig. 3. Representation of the triangles $\mathcal{T}_{k}$

On $\mathcal{T}_{k_{0}} \cap \mathcal{T}_{0}$, equations (123) and (124) can be simply rewritten, for $l=1 \ldots n+m$ as

$$
\epsilon_{l} \partial_{x} F_{l}(x, \xi)+\nu_{l} \partial_{\xi} F_{l}(x, \xi)=\Sigma_{l}(x, \xi) F(x, \xi)
$$

where $F=\left(K_{i, 1} \ldots K_{i, n}, L_{i, 1}, \ldots L_{i, m}\right)^{T}$. The functions $\epsilon_{l}$ and $\nu_{l}$ are defined according to the location of the boundary condition by

$$
\begin{aligned}
& \epsilon_{l}= \begin{cases}-\lambda_{i} & \text { if } l \leq n \\
-\lambda_{i} & \text { if } l>n \text { and } l-n<k_{0} \\
+\lambda_{i} & \text { else }\end{cases} \\
& \nu_{l}= \begin{cases}-\lambda_{l} & \text { if } l \leq n \\
+\mu_{l} & \text { if } l>n \text { and } l-n<k_{0} \\
-\mu_{l} & \text { else }\end{cases}
\end{aligned}
$$

The homogeneous system, obtained by taking $\Sigma_{l}(x, \xi)=0$ along with the corresponding boundary conditions is wellposed. If we choose $\alpha_{k_{0}}$ such that

$$
\frac{\mu_{k_{0}}-1}{\lambda_{i}}<\alpha_{k_{0}}=\frac{\mu_{k_{0}-1}+\mu_{k_{0}}}{2 \lambda_{i}}<\frac{\mu_{k_{0}}}{\lambda_{i}}
$$

we easily get

$$
\alpha_{k_{0}} \epsilon_{l}+\nu_{l}=\left\{\begin{array}{l}
\frac{-\mu_{k_{0}-1}-\mu_{k_{0}}}{2}-\lambda_{l} \text { if } l \leq n \\
\frac{-\mu_{k_{0}-1}-\mu_{k_{0}}}{2}+\mu_{l} \text { if } l>n \text { and } l-n<k_{0} \\
\frac{+\mu_{k_{0}-1}+\mu_{k_{0}}}{2}-\mu_{l} \text { else }
\end{array}\right.
$$

In the first case, the result is always negative. If $l-n<k_{0}$, $\mu_{l}<\mu_{k_{0}}<\frac{\mu_{k_{0}}+\mu_{k_{0}-1}}{2}$. Consequently, for the second case the result is still negative. The same holds for the third case. Consequently, the two hypothesis of Theorem 3 are verified and we can conclude to the well-posedness of the kernel equations on $\mathcal{T}_{k_{0}}$. This concludes the initialization.

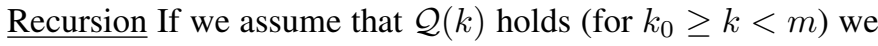


can easily prove using Theorem 3 that $\mathcal{Q}(k+1)$ holds. The well-posedness of the homogeneous system is direct using $\mathcal{Q}(k)$ and one can easily check that the second hypothesis of the theorem holds choosing $\alpha_{k+1}$ :

$$
\alpha_{k+1}=\frac{\mu_{k+1}+\mu_{k}}{2}
$$

with the convention $\mu_{m+1}=\mu_{m}+1$. Moreover this iteration provides us the boundary condition for the next triangle. This concludes the proof.

Remark 11: If $a_{i 1}<\bar{a}$, the previous result still holds taking $a_{i 0}=\bar{a}$

This proves the well-posedness of the $i^{\text {th }}$ line of the kernels $K$ and $J$ on $\mathcal{T}_{0}$. Consequently $P(q)$ is true and the well-posedness of the kernels $K, L, \bar{K}, \bar{L}$ on $\mathcal{T}_{0}$ is proved.

\section{INVERTIBILITY OF THE FREDHOLM TRANSFORMATION}

Unlike the Volterra transformation, the Fredholm transformation is not always invertible. In [11], the authors prove the invertibility of such a transformation in the case of a first-order integro-differential hyperbolic equation. In [6] the invertibility of the transformation is proved in the scalar case. In this section we use similar arguments (in particular we rely on the Fredholm alternative) to prove the invertibility of our transformation.

1) Operator formulation of the Fredholm transformation and properties

In this subsection we rewrite the previous Fredholm transformation using operators. This will lead to some relations verified by the adjoint operators. The Fredholm transformation (57)-(58) can be written as an operator $P$ acting on $\left(\begin{array}{l}u \\ v\end{array}\right)$. More precisely we have

$$
\begin{aligned}
P & =I d-Q \\
\left(\begin{array}{l}
\alpha \\
\beta
\end{array}\right) & =P\left(\begin{array}{l}
u \\
v
\end{array}\right)
\end{aligned}
$$

where $Q:\left(L^{2}(0,1)\right)^{n+m} \rightarrow\left(L^{2}(0,1)\right)^{n+m}$ is the integral operator defined by

$$
Q\left(\begin{array}{l}
u \\
v
\end{array}\right)=\int_{0}^{1}\left(\begin{array}{l}
Q_{11}(x, \xi) u(t, \xi)+Q_{12}(x, \xi) v(t, \xi) \\
Q_{21}(x, \xi) u(t, \xi)+Q_{22}(x, \xi) v(t, \xi)
\end{array}\right) d \xi
$$

Its adjoint is:

$$
Q^{*}\left(\begin{array}{l}
u \\
v
\end{array}\right)=\int_{0}^{1}\left(\begin{array}{l}
Q_{11}(\xi, x) u(t, \xi)+Q_{21}(\xi, x) v(t, \xi) \\
Q_{12}(\xi, x) u(t, \xi)+Q_{22}(\xi, x) v(t, \xi)
\end{array}\right) d \xi
$$

One can easily check that:

$$
Q^{*}\left(D\left(A^{*}\right)\right) \subset D\left(A^{*}\right)
$$

The control $\left(\begin{array}{l}U \\ V\end{array}\right)$ can also be rewritten using operators

$$
\left(\begin{array}{l}
U \\
V
\end{array}\right)=\Gamma\left(\begin{array}{l}
u \\
v
\end{array}\right)
$$

with

$$
\Gamma\left(\begin{array}{l}
u \\
v
\end{array}\right)=\int_{0}^{1}\left(\begin{array}{l}
Q_{11}(0, \xi) u(t, \xi)+Q_{12}(0, \xi) v(t, \xi) \\
Q_{21}(1, \xi) u(t, \xi)+Q_{22}(1, \xi) v(t, \xi)
\end{array}\right) d \xi
$$

Using (47) and (135) yields

$$
\frac{d}{d t}\left(\begin{array}{l}
\alpha \\
\beta
\end{array}\right)=A_{0}\left(\begin{array}{l}
\alpha \\
\beta
\end{array}\right)=A_{0} P\left(\begin{array}{l}
u \\
v
\end{array}\right)
$$

Moreover using (11) and (135) we get

$$
\begin{aligned}
\frac{d}{d t}\left(\begin{array}{l}
\alpha \\
\beta
\end{array}\right) & =\frac{d}{d t}\left(P\left(\begin{array}{l}
u \\
v
\end{array}\right)\right) \\
& =P A\left(\begin{array}{l}
u \\
v
\end{array}\right)+P B \Gamma\left(\begin{array}{l}
u \\
v
\end{array}\right)
\end{aligned}
$$

Consequently $P$ and $\Gamma$ satisfy the following relation:

$$
A_{0} P=P A+P B \Gamma
$$

Taking the adjoints, this is equivalent to

$$
P^{*} A_{0}^{*}=A^{*} P^{*}+\Gamma^{*} B^{*} P^{*}
$$

\section{2) The Fredholm alternative}

We give first the following useful lemmas:

Lemma 7: $\operatorname{ker} P^{*} \subset D\left(A_{0}^{*}\right)=D\left(A^{*}\right)$

Proof: Let us consider $z \in \operatorname{ker} P^{*}$. Consequently we have $P^{*} z=0$. We can rewrite it

$$
\left(\begin{array}{l}
z_{1} \\
z_{2}
\end{array}\right)=\int_{0}^{1}\left(\begin{array}{l}
Q_{11}(\xi, x) z_{1}(t, \xi)+Q_{21}(\xi, x) z_{2}(t, \xi) \\
Q_{12}(\xi, x) z_{1}(t, \xi)+Q_{22}(\xi, x) z_{2}(t, \xi)
\end{array}\right) d \xi
$$

If we evaluate the first line for $x=1$ and the second one for $x=0$, using the fact that $Q_{11}(\xi, 0)=Q_{21}(\xi, 0)=$ $Q_{12}(\xi, 1)=Q_{22}(\xi, 1)=0$, we get

$$
z_{1}(1)=z_{2}(0)=0
$$

Consequently $z \in D\left(A_{0}^{*}\right)$ and we can write

$$
\text { ker } P^{*} \subset D\left(A_{0}^{*}\right)
$$

Lemma 8: $\operatorname{ker} P^{*} \subset$ ker $B^{*}$

Proof: Let us consider $z \in \operatorname{ker} P^{*}$. Consequently we have $P^{*} z=0$. We can rewrite it

$$
\left(\begin{array}{l}
z_{1} \\
z_{2}
\end{array}\right)=\int_{0}^{1}\left(\begin{array}{l}
Q_{11}(\xi, x) z_{1}(t, \xi)+Q_{21}(\xi, x) z_{2}(t, \xi) \\
Q_{12}(\xi, x) z_{1}(t, \xi)+Q_{22}(\xi, x) z_{2}(t, \xi)
\end{array}\right) d \xi
$$

If we evaluate the first line for $x=0$ and the second one for $x=1$, using the fact that $Q_{11}(\xi, 1)=Q_{21}(\xi, 1)=$ $Q_{12}(\xi, 0)=Q_{22}(\xi, 0)=0$, we get

$$
z_{1}(0)=z_{2}(1)=0
$$

Consequently $z \in \operatorname{ker} B^{*}$ and we can write

$$
\operatorname{ker} P^{*} \subset \operatorname{ker} B^{*}
$$

Lemma 9: $\forall \lambda \in \Re \operatorname{ker}\left(\lambda I d-A_{0}^{*}\right) \cap \operatorname{ker} B^{*}=\{0\}$ 
Proof: Let us consider $\nu \in \Re$ and $z \in \operatorname{ker}\left(\nu I d-A_{0}^{*}\right) \cap$ ker $B^{*}=\{0\}$. Consequently we have

$0=\left(\begin{array}{c}z_{1}(t, x)_{x}^{T} \Lambda^{+}+z_{1}(t, x)^{T} \Omega+z_{2}(t, x)^{T} \bar{\Gamma}-\nu z_{1}(t, x)^{T} \\ -z_{2}(t, x)_{x}^{T} \Lambda^{-}+z_{2}(t, x)^{T} \bar{\Omega}+z_{1}(t, x)^{T} \Gamma-\nu z_{2}(t, x)^{T}\end{array}\right)$

with the boundary conditions

$$
z_{1}(0)=z_{2}(0)=0
$$

Consequently (using the Cauchy-Lipschitz' theorem) we have $z=\left(\begin{array}{l}0 \\ 0\end{array}\right)$

We can now state the following theorem

Theorem 5: The map $P^{*}=I d-Q^{*}$ is invertible

Proof: Since $Q^{*}$ is a compact operator we can use the Fredholm alternative (e.g [9]): $I d_{2}-Q^{*}$ is either non-injective or surjective. Consequently it suffices to prove that $P^{*}$ is injective. In addition, the Fredholm alternative also gives [9]

$$
\operatorname{dim} \operatorname{ker}\left(I d-Q^{*}\right)<+\infty
$$

By contradiction we assume that ker $P^{*} \neq\{0\}$. We first prove that ker $P^{*}$ is stable by $A_{0}^{*}$. We have $\operatorname{ker} P^{*} \subset D\left(A_{0}^{*}\right)$. Let then consider $z \in \operatorname{ker} P^{*}$. Using (144) we can obtain

$$
P^{*} A_{0}^{*} z=0
$$

We thus have $A_{0}^{*} z \in \operatorname{ker} P^{*}$. Consequently the restriction $A_{0_{\mid k e r}^{*} P^{*}}^{*}$ of $A_{0}^{*}$ to ker $P^{*}$ is a linear operator from ker $P^{*}$ to $\operatorname{ker} P^{*}$. Since the dimension of $\operatorname{ker} P^{*}$ is finite we can find at least one eigenvalue $\nu$. Let $e \in \operatorname{ker} P^{*}$ be a corresponding eigenvector (by definition $e \neq 0$ ). We have $e \in \operatorname{ker} P^{*}$ and so $e \in \operatorname{ker} B^{*}$. Moreover we have $A_{0}^{*} e=\nu e$. Consequently

$$
e \in \operatorname{ker}\left(\nu I d-A_{0}^{*}\right) \cap \operatorname{ker} B^{*}
$$

which contradicts Lemma 9 and concludes the proof.

\section{CONTROL LAW AND MAIN RESUlTS}

We now state the main stabilization result as follows:

Theorem 6: System (1)-(2) with the following feedback control laws

$$
\begin{gathered}
U(t)=-\int_{0}^{1}(K(0, \xi) u(t, \xi)+L(0, \xi) v(t, \xi)) d \xi \\
V(t)=-\int_{0}^{1}(\bar{M}(1, \xi) u(t, \xi)+\bar{N}(1, \xi) v(t, \xi)) d \xi
\end{gathered}
$$

where $K, L$ and $\bar{M}, \bar{N}$ are defined by (65)-(70) and (83)-(88), reaches its zero equilibrium in finite time $t_{F}$, where $t_{F}$ is given by (18). The zero equilibrium is exponentially stable in the $L^{2}$-sense.

Proof: Notice that evaluating (52) at $x=0$ yields (156) and evaluating (53) at $x=1$ yields (157). Since the kernels are invertible, there exists a unique operator $\mathcal{S}$ such that

$$
\left(\begin{array}{l}
u \\
v
\end{array}\right)=\mathcal{S}\left(\begin{array}{l}
\alpha \\
\beta
\end{array}\right)
$$

Applying Lemma 2 implies that $(\alpha, \beta)$ go to zero in finite time $t_{F}$, therefore $(u, v)$ converge to zero in finite time $t_{F}$

\section{OBSERVER DESIGN AND OUTPUT FEEDBACK CONTROLLER}

In this section we design an observer that relies on the measurements of $u$ at the right boundary and $v$ at the left boundary, i.e we measure

$$
\begin{aligned}
& y_{1}(t)=u(t, 1) \\
& y_{2}(t)=v(t, 0)
\end{aligned}
$$

Then, using the estimates given by our observer and the control law (156)-(157), we derive an output feedback controller.

\section{A. Observer design}

The observer equations reads as follows

$$
\begin{aligned}
& \hat{u}_{t}(t, x)+\Lambda^{+} \hat{u}_{x}(t, x)=\Sigma^{++} \hat{u}(t, x)+\Sigma^{+-} \hat{v}(t, x) \\
& -P_{11}(x)(\hat{u}(t, 1)-u(t, 1))-P_{12}(\hat{v}(t, 0)-v(t, 0)) \\
& \hat{v}_{t}(t, x)-\Lambda^{-} \hat{v}_{x}(t, x)=\Sigma^{-+} \hat{u}(t, x)+\Sigma^{--} \hat{v}(t, x) \\
& -P_{21}(x)(\hat{u}(t, 1)-u(t, 1))-P_{22}(\hat{v}(t, 0)-v(t, 0))
\end{aligned}
$$

with the boundary conditions

$$
\hat{u}(t, 0)=U(t), \quad \hat{v}(t, 1)=V(t)
$$

where $P_{11}(\cdot), P_{21}(\cdot), P_{12}(\cdot)$ and $P_{22}(\cdot)$ have yet to be designed. This yield the following error system

$$
\begin{aligned}
\tilde{u}_{t}(t, x) & +\Lambda^{+} \tilde{u}_{x}(t, x)=\Sigma^{++} \tilde{u}(t, x)+\Sigma^{+-} \tilde{v}(t, x) \\
& -P_{11}(x) \tilde{u}(t, 1)-P_{12}(x) \tilde{v}(t, 0) \\
\tilde{v}_{t}(t, x) & -\Lambda^{-} \tilde{v}_{x}(t, x)=\Sigma^{-+} \tilde{u}(t, x)+\Sigma^{--} \tilde{v}(t, x) \\
& -P_{21}(x) \tilde{u}(t, 1)-P_{22}(x) \tilde{v}(t, 0)
\end{aligned}
$$

with the boundary conditions

$$
\tilde{u}(t, 0)=0, \quad \tilde{v}(t, 1)=0
$$

This system evolves in $[0, T] \times[0, x]$ and its initial condition $(\tilde{u}(0, x), \tilde{v}(0, x))=\left(\tilde{u}_{0}(x), \tilde{v}_{0}(x)\right)$ belongs to $L^{2}([0,1])$. We define the following system (which is the same as above changing $t$ in $T-t$ ).

$$
-\frac{d}{d t}\left(\begin{array}{l}
\bar{u} \\
\bar{v}
\end{array}\right)=\bar{A}\left(\begin{array}{l}
\bar{u} \\
\bar{v}
\end{array}\right)
$$

evolving in $[0, T] \times[0, x]$ with the $L^{2}([0,1])$ arbitrary initial condition

$$
\left(\begin{array}{l}
\bar{u}(0, x) \\
\bar{v}(0, x)
\end{array}\right)=\left(\begin{array}{l}
\bar{u}_{0}(x) \\
\bar{v}_{0}(x)
\end{array}\right)
$$

The operator $\bar{A}$ is thus defined by

$$
\begin{gathered}
\bar{A}: D(\bar{A}) \subset\left(L^{2}(0,1)\right)^{n+m} \rightarrow\left(L^{2}(0,1)\right)^{n+m} \\
\left(\begin{array}{c}
\bar{u} \\
\bar{v}
\end{array}\right) \longmapsto\left(\begin{array}{c}
-\Lambda^{+} \bar{u}_{x}+\Sigma^{++} \bar{u}+\Sigma^{+-} \bar{v} \\
\Lambda^{-} \bar{v}_{x}+\Sigma^{-+} \bar{u}+\Sigma^{--} \bar{v}
\end{array}\right) \\
+\left(\begin{array}{l}
-P_{11}(x) \bar{u}(t, 1)-P_{12}(x) \bar{v}(t, 0) \\
-P_{21}(x) \bar{u}(t, 1)-P_{22}(x) \bar{v}(t, 0)
\end{array}\right)
\end{gathered}
$$

with

$$
D(\bar{A})=\left\{(u, v) \in\left(H^{1}(0,1)\right)^{n+m} \mid \bar{u}(0)=\bar{v}(1)=0\right\}
$$




\section{B. A dual system}

In order to find the observer gains we define a control problem that is the dual of the observer problem. The observer gains will then be defined by the gains of the dual controller.

1) A new control problem

Let us consider the following system

$$
\begin{aligned}
& \phi_{t}(t, x)-\Lambda^{+} \phi_{x}(t, x)=\left(\Sigma^{-+}\right)^{T} \psi(t, x)+\left(\Sigma^{++}\right)^{T} \phi(t, x) \\
& \psi_{t}(t, x)+\Lambda^{-} \psi_{x}(t, x)=\left(\Sigma^{--}\right)^{T} \psi(t, x)+\left(\Sigma^{+-}\right)^{T} \phi(t, x)
\end{aligned}
$$

evolving in $\{(t, x) \mid t>0, \quad x \in[0,1]\}$, with the following linear boundary conditions

$$
\psi(t, 0)=U(t), \quad \phi(t, 1)=V(t)
$$

and the arbitrary initial conditions (belonging to $L^{2}([0,1])$ )

$$
\phi(0, x)=\phi_{0}(x), \quad \psi(0, x)=\psi_{0}(x)
$$

Using Theorem 6, we can explicitly compute kernels $K_{1}, L_{1}, \bar{M}_{1}$ and $\bar{N}_{1}$ such that system (172)-(173) with the following feedback law

$$
\begin{aligned}
& U(t)=-\int_{0}^{1}\left(K_{1}(0, \xi) \phi(t, \xi)+L_{1}(0, \xi) \psi(t, \xi)\right) d \xi \\
& V(t)=-\int_{0}^{1}\left(\bar{M}_{1}(1, \xi) \phi(t, \xi)+\bar{N}_{1}(1, \xi) \psi(t, \xi)\right) d \xi
\end{aligned}
$$

reaches its zero equilibrium in time $t_{F}$ where $t_{F}$ is given by (18). As seen in Section II-B, this system can be rewritten in the abstract form

$$
\frac{d}{d t}\left(\begin{array}{l}
\phi \\
\psi
\end{array}\right)=A\left(\begin{array}{l}
\phi \\
\psi
\end{array}\right)+B\left(\begin{array}{l}
U \\
V
\end{array}\right)
$$

with the initial condition

$$
\left(\begin{array}{l}
\phi(0, x) \\
\psi(0, x)
\end{array}\right)=\left(\begin{array}{l}
\phi_{0}(x) \\
\psi_{0}(x)
\end{array}\right)
$$

where $\mathrm{A}$ and $\mathrm{B}$ are defined in a similar form as the one presented in equations (12)-(17).

2) Definition of the observer gains

We now define the gains of the observer as

$$
\begin{array}{ll}
P_{11}(x)=\bar{M}_{1}(1, x)^{T} \Lambda^{+} & P_{21}(x)=\bar{N}_{1}(1, x)^{T} \Lambda^{+} \\
P_{12}(x)=K_{1}(0, x)^{T} \Lambda^{-} & P_{22}(x)=L_{1}(0, x)^{T} \Lambda^{-}
\end{array}
$$

Theorem 7: System (168)-(169) where $P_{11}, P_{12}, P_{21}$ and $P_{22}$ are defined by (180)-(181) is the adjoint of system (178)(179). Moreover, both system reach their zero equilibrium in time $t_{F}$ where $t_{F}$ is defined by (18).

Proof: We define $\langle\cdot, \cdot\rangle$ as the scalar product associated to the $L^{2}$-norm. For every solution $(\tilde{u}, \tilde{v})$ of (168)-(169) and every solution $(\phi, \psi)$ of (178)-(179) (with any initial conditions) we have

$$
<\frac{d}{d t}\left(\begin{array}{l}
\phi \\
\psi
\end{array}\right)-A\left(\begin{array}{l}
\phi \\
\psi
\end{array}\right)-B\left(\begin{array}{l}
V \\
U
\end{array}\right),\left(\begin{array}{l}
\tilde{u} \\
\tilde{v}
\end{array}\right)>=0
$$

It yields

$$
\begin{aligned}
0 & =\int_{0}^{\tau} \int_{0}^{1}\left(-\bar{u}_{t}^{T}(t, x)+\bar{u}_{x}^{T}(t, x) \Lambda^{+}-\bar{u}^{T}(t, x)\left(\Sigma^{++}\right)^{T}\right. \\
& \left.-\bar{v}(t, x)^{T}\left(\Sigma^{+-}\right)^{T}\right) \phi(t, x)+\left(-\bar{v}_{t}^{T}(t, x)-\bar{v}_{x}^{T}(t, x) \Lambda^{-}\right. \\
& \left.-\bar{u}(t, x)\left(\Sigma^{-+}\right)^{T}-\bar{v}(t, x)\left(\Sigma^{--}\right)^{T}\right) \psi(t, x) d x d t \\
& +\int_{0}^{1}\left(\bar{v}^{T}(\tau, x) \psi(\tau, x)-\bar{v}^{T}(0, x) \psi(0, x)\right. \\
& \left.+\bar{u}^{T}(\tau, x) \phi(\tau, x)-\bar{u}^{T}(0, x) \phi(0, x)\right) d x \\
& +\int_{0}^{\tau} \int_{0}^{1}\left(\bar{u}(t, 1)^{T} \Lambda^{+}\left(\bar{M}_{1}(1, x) \phi(t, x)+\bar{N}_{1}(1, x) \psi(t, x)\right)\right. \\
& \left.+\bar{v}(t, 0)^{T} \Lambda^{-}\left(K_{1}(0, x) \phi(t, x)+L_{1}(0, x) \psi(t, x)\right)\right) d t
\end{aligned}
$$

Using this expression, it can easily be seen that system (168)(169) is the adjoint of system (178)-(179). Moreover, taking $\tau=t_{F}$ yields

$$
\begin{aligned}
0 & =<\left(\begin{array}{c}
\phi \\
\psi
\end{array}\right),-\frac{d}{d t}\left(\begin{array}{c}
\bar{u} \\
\bar{v}
\end{array}\right)-\bar{A}\left(\begin{array}{l}
\bar{u} \\
\bar{v}
\end{array}\right)> \\
& +\int_{0}^{1}-\bar{u}^{T}(0, x) \phi(0, x)-\bar{v}^{T}(0, x) \psi(0, x) d x \\
\Rightarrow 0 & =\bar{u}^{T}(0, x) \phi(0, x)+\bar{v}^{T}(0, x) \psi(0, x)
\end{aligned}
$$

Since this has to be true for any initial condition, it implies that

$$
\bar{v}(0, x)=0, \quad \bar{u}(0, x)=0
$$

Consequently using the change of variable $t=t_{F}-t$ yields

$$
\tilde{v}\left(t_{F}, x\right)=0, \quad \tilde{u}\left(t_{F}, x\right)=0
$$

\section{Output feedback controller}

The estimates can be used in a observer-controller to derive an output feedback law yielding finite-time stability of the zero equilibrium

Lemma 10: Consider the system composed of (1)-(3) and target system (162)-(164) with the following control law

$$
\begin{gathered}
U(t)=-\int_{0}^{1}(K(0, \xi) \hat{u}(t, \xi)+L(0, \xi) \hat{v}(t, \xi)) d \xi \\
V(t)=-\int_{0}^{1}(\bar{M}(1, \xi) \hat{u}(t, \xi)+\bar{N}(1, \xi) \hat{v}(t, \xi)) d \xi
\end{gathered}
$$

where $K, L$ and $\bar{M}, \bar{N}$ are defined by (65)-(70) and (83)-(88). Its solutions $(u, v, \hat{u}, \hat{v})$ converge in finite time to zero

Proof: The convergence of the observer error states $\tilde{u}, \tilde{v}$ to zero for $t_{F} \leq t$ is ensured by Theorem 7 , along with the existence of the backstepping transformation. Thus, once $t_{F} \leq$ $t, v(t, 0)=\hat{v}(t, 0)$ and one can use Theorem 6 . Therefore for $2 t_{F} \leq t$, one has $(\tilde{u}, \tilde{v}, \hat{u}, \hat{v}) \equiv 0$ which yields $(u, v) \equiv 0$.

\section{APPliCATION: STATE ESTIMATION DURING UNDERBALANCED DRILLING}

In this section, we illustrate the benefits of our approach by applying it to an industrial problem. 


\section{A. Problem description}

Consider the drilling system schematically depicted on Figure 4. It consists of a 2530 meter-long drillpipe, rotating

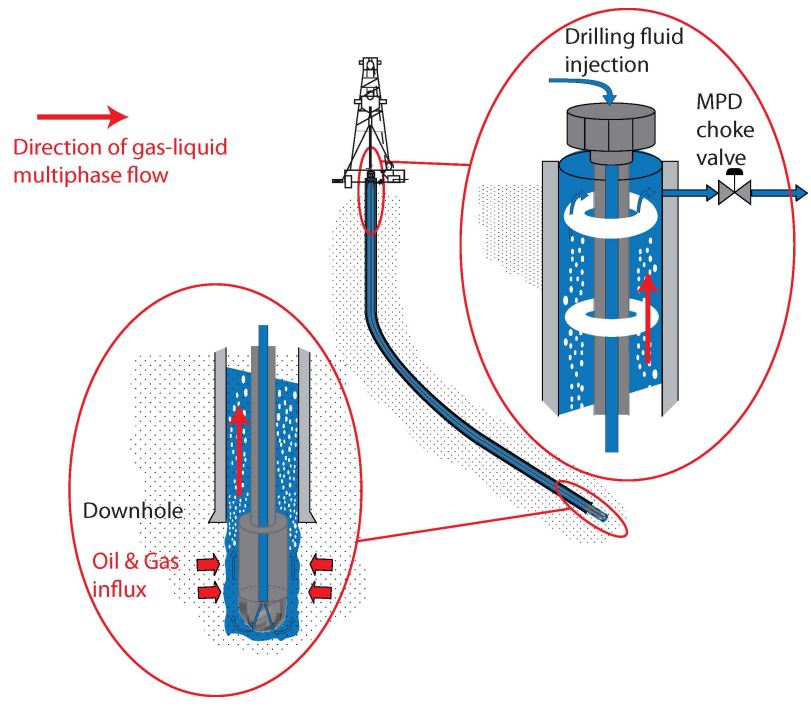

Fig. 4. Schematic view of drilling facilities. In UnderBalanced Drilling (UBD), oil and gas enter the annulus as the drilling process goes on.

around its main axis, through which is injected a drilling fluid, typically water-based mud. At the end of the pipe, the fluid exits through the drillbit (which chatters and cuts the rock) and circulates back to the surface inside the annulus, carrying rock cuttings. For numerous reasons [1], it is desirable to produce oil and gas from the reservoir as the drilling process goes on when possible, a technique referred to as UnderBalanced Drilling (UBD). The term underbalanced refers to the value of the pressure at the bottom of the annulus, which must be lower than the value of the pressure of hydrocarbons inside the reservoir (called the balance point) for the oil and gas to flow in. To ensure safety and efficiency of operations, it is desirable to monitor at all time the amount of gas inside the well. This is a difficult task since sensors cannot be placed all along the drillpipe, and the dynamics of multiphase flow are known to be complex [3]. The presence of gas, in particular, makes the distributed, delay-like nature of the dynamics predominant and may generate instabilities, such as severe slugging.

\section{B. Observer design}

The simulation model is a Drift-Flux Model (DFM) described in [2]. It models the flow of liquid (oil, water and drilling fluid being considered as one liquid phase) and gas along the drillstring using two mas conservation laws and one combined momentum conservation law. Along with closure relations, this yields a set of three nonlinear transport PDEs with appropriate boundary conditions. The model can be linearized around a given equilibrium profile, which yields a system of the form (1)-(3) with $n=2$ and $m=1$ (see, e.g., [3]).

The model used to design the observer is the same model, starting from a different initial condition. This choice is debatable, since perfect knowledge of the model is unrealistic,
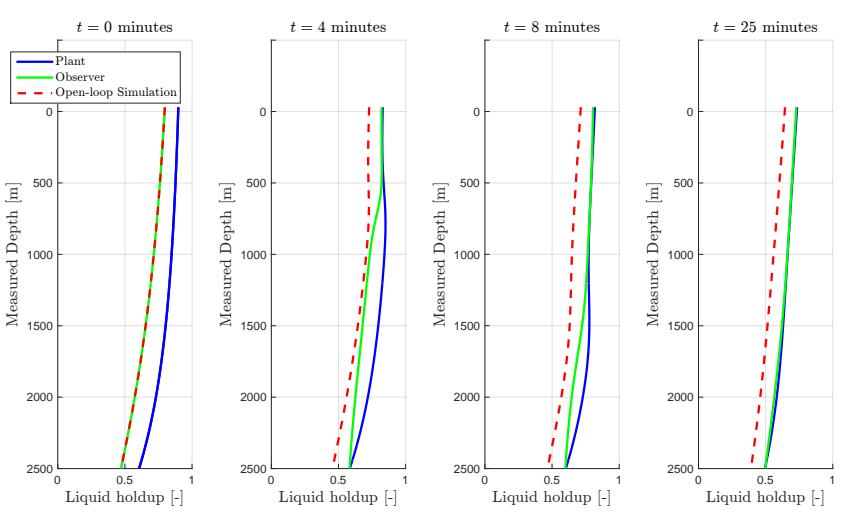

Fig. 5. Snapshots of the spatial profile of the liquid holdup as a function of depth. The profile of the observer converges to the true profile in approximately 25 minutes.

however assessing the robustness of the model with respect to model uncertainty is out of the scope of this paper and will be the topic of future contributions. Notice that the nonlinearity of the model, combined with the offset in the initial condition already yields differences in the linearized model parameters.

Similarly to [16], the observer is designed by copying the nonlinear equations and adding the linear output error correction terms such that linearizing the observer equations yields (161)-(164).

\section{Simulation results}

Figure 5 depicts snapshots of the volume fraction of liquid (holdup), as a function of well depth at different time instants of a transient simulation. The three curves respectively correspond to the 'plant', the observer and an open-loop simulation starting from the same initial condition as the observer, without any correction terms. The holdup profile of the observer converges in approximately 25 minutes to the plant profile, while the open-loop profile would take around two hours (the settling time of this particular system) to converge. This plot is not shown here due to lack of space.

\section{CONCLUding REMARK}

Using a backstepping approach we have presented a stabilizating boundary feedback law for a general class of linear first-order system controlled in both boundaries. Moreover, the zero equilibrium of the system is reached in minimum time $t_{F}$ which is the largest time between the two transport times in each direction.

Besides, the presented result narrows the gap with the theoretical controllability results of [20]. These results, although they do not provide explicit control law, ensure exact minimumtime controllability with less control inputs than what is currently achievable using backstepping. More generally, this raises the question of the links between controllability and stabilization by backstepping. In particular future works will consider first-order systems with a smaller number of controls in each boundary. 


\section{APPENDIX}

In this appendix, we prove Theorem 3. Assume that the assumptions of Theorem 3 hold then the system (89) with boundary conditions (91) has an unique solution $F \in L^{\infty}(\mathcal{D})$. Classically (see [19] and [23]), the proof follows three steps:

- First, we compute the characteristic lines

- In each domain the equations are transformed into integral equations.

- Finally, a method of successive approximations is used to find a solution to the integral equations.

\section{A. Transformation into integral equations}

The first assumption of Theorem 3 yields the existence and uniqueness of characteristic curves defined as follows.

For each $1 \leq j \leq n+m$ and $(x, \xi) \in \mathcal{D}$, we now define the following characteristic lines $\left(x_{j}(x, \xi, \cdot), \xi_{j}(x, \xi, \cdot)\right)$ corresponding to equation (89)

$$
\begin{aligned}
& \left\{\begin{array}{l}
\frac{d x_{j}}{d s}(x, \xi, s)=\epsilon_{j} \quad s \in\left[0, s_{j}^{F}(x, \xi)\right] \\
x_{j}(x, \xi, 0)=x_{j}^{0}(x, \xi), \quad x_{j}\left(x, \xi, s_{j}^{F}(x, \xi)\right)=x
\end{array}\right. \\
& \left\{\begin{array}{c}
\frac{d \xi_{j}}{d s}(x, \xi, s)=\nu_{j} \quad s \in\left[0, s_{j}^{F}(x, \xi)\right] \\
\xi_{j}(x, \xi, 0)=\xi_{j}^{0}(x, \xi), \quad \xi_{j}\left(x, \xi, s_{j}^{F}(x, \xi)\right)=\xi
\end{array}\right.
\end{aligned}
$$

These lines originate at the point $\left(x_{j}^{0}(x, \xi), \xi_{j}^{0}(x, \xi)\right)$ located on the boundary of the domain and terminate on $(x, \xi)$.

Integrating (89) along these characteristics and using boundary conditions (91) yields

$$
\begin{aligned}
F_{j}(x, \xi) & =f_{j}\left(M_{j}^{0}(x, \xi, s)\right)+ \\
& \int_{0}^{s_{j}^{F}(x, \xi)} \Sigma_{j}\left(M_{j}(x, \xi, s)\right) F\left(M_{j}(x, \xi, s)\right) d s
\end{aligned}
$$

where we denote $M_{j}(x, \xi, s)=\left(x_{j}(x, \xi, s), \xi_{j}(x, \xi, s)\right)$ and $M_{j}^{0}(x, \xi, s)=\left(x_{j}^{0}(x, \xi, s), \xi_{j}^{0}(x, \xi, s)\right)$

\section{B. Method of successive approximations}

In order to solve (192) we use the method of successive approximations. We define

$$
\Phi_{j}[F](x, \xi)=\int_{0}^{s_{j}^{F}(x, \xi)} \Sigma_{j}\left(M_{j}\left(x, \xi, s_{j}\right)\right) F\left(M_{j}\left(x, \xi, s_{j}\right)\right) d s
$$

We now construct the sequence $F^{p}$ defined by

$$
\begin{aligned}
F^{0}(x, \xi) & =0 \\
F^{p+1}(x, \xi) & =\left(\begin{array}{c}
f_{1}\left(M_{1}^{0}\left(x, \xi, s_{j}\right)\right) \\
\vdots \\
f_{n+m}\left(M_{(n+m)}^{0}\left(x, \xi, s_{j}\right)\right)
\end{array}\right) \\
& +\left(\begin{array}{c}
\Phi_{1}\left[F^{p}\right](x, \xi) \\
\vdots \\
\Phi_{(n+m)}\left[F^{p}\right](x, \xi)
\end{array}\right)
\end{aligned}
$$

Consequently, if the sequence $F^{p}$ has a limit, then this limit is a solution of the integral equation and therefore of the original system.
We define the increment $\Delta F^{p}=F^{p}-F^{p-1}$. Provided the limit exists one has

$$
F(x, \xi)=\lim _{p \rightarrow+\infty} F^{p}(x, \xi)=\sum_{p=0}^{+\infty} \Delta F^{p}(x, \xi)
$$

We now prove the convergence of the series.

\section{Convergence of the successive approximations series}

The proof of convergence is based on the following Lemmas.

Lemma 11: Assume that (92) holds. then for all $j=$ $1 \ldots n+1,(x, \xi) \in \mathcal{D}$, the following function is strictly increasing

$$
\begin{aligned}
\phi_{x, \xi}: s \in\left[0, s_{j}^{F}(x, \xi)\right] \longmapsto & -\alpha x_{j}(x, \xi, s)-\xi_{j}(x, \xi, s) \\
& +(\alpha+1)
\end{aligned}
$$

Proof: The proof is trivial. Recalling (92) yields

$$
\phi_{x, \xi}^{\prime}(s)=-\alpha \epsilon_{j}-\nu_{j}>0
$$

This concludes the proof.

Lemma 12: For all $j=1 \ldots n+m$ the following inequalities hold

$$
\begin{aligned}
\int_{0}^{s_{j}^{F}(x, \xi)} & \left(-\alpha x_{i j}(x, \xi, s)-\xi_{i j}(x, \xi, s)+(\alpha+1)\right)^{p} d s \\
& \leq \frac{1}{\delta} \frac{((\alpha+1)-\alpha x-\xi)^{p+1}}{p+1}
\end{aligned}
$$

Proof: Consider the following change of variables

$$
\tau=\phi_{x, \xi}(s)
$$

It yields

$$
\begin{aligned}
& \int_{0}^{s_{j}^{F}(x, \xi)}\left(-\alpha x_{j}(x, \xi, s)-\xi_{j}(x, \xi, s)+(\alpha+1)\right)^{p} d s \\
= & \int_{-\alpha x_{j}^{0}(x, \xi)-\xi_{j}^{0}(x, \xi)+(\alpha+1)}^{-\alpha x-\xi+(\alpha+1)} \frac{\tau^{p} d \tau}{-\alpha \epsilon_{j}-\nu_{j}}
\end{aligned}
$$

Using (92) and the definition of $\delta$, this yields

$$
\begin{aligned}
& \int_{0}^{s_{j}^{F}(x, \xi)}\left(\alpha x_{j}(x, \xi, s)+\xi_{j}(x, \xi, s)\right)^{p} d s \\
< & \frac{(-\alpha x-\xi+(\alpha+1))^{p+1}}{\delta(p+1)} \\
- & \frac{\left(-\alpha x_{j}^{0}(x, \xi)-\xi_{j}^{0}(x, \xi)+(\alpha+1)\right)^{p+1}}{\delta(p+1)}
\end{aligned}
$$

Since $\left(x_{j}^{0}(x, \xi), \xi_{j}^{0}(x, \xi)\right) \in \mathcal{D}$ and since $\alpha>0$, one has $-\alpha x_{j}^{0}(x, \xi)-\xi_{j}^{0}(x, \xi)+\alpha+1 \geq 0$ which yields the result.

Lemma 13: Let $M>0$ be such that

$$
M>\bar{\Sigma} \delta
$$

where $\bar{\Sigma}$ is defined as

$$
\bar{\Sigma}=\max _{(x, \xi) \in \mathcal{D}} \max _{\|F\| \neq 0} \frac{\|\Sigma(x, \xi) F\|}{\|F\|}
$$


If for some $1 \leq q$ and some $\bar{f}>0$ one has, for all $(x, \xi) \in \mathcal{T}_{0}$

$\forall j=1 \ldots m+n \quad\left|\Delta F_{j}^{p}(x, \xi)\right| \leq \frac{M^{p}(-\alpha x-\xi+(\alpha+1))^{p}}{p !}$

Then one has $\forall j=1 \ldots m+n$

$$
\left|\Delta F_{j}^{p+1}(x, \xi)\right| \leq \frac{M^{p+1}(-\alpha x-\xi+(\alpha+1))^{p+1}}{(p+1) !}
$$

Proof: Assume that (205) holds for some fixed $1 \leq p$. Let us consider $1 \leq j \leq(m+n)$.

$$
\begin{aligned}
& \left|\Delta F_{j}^{p+1}(x, \xi)\right|=\left|\Phi_{j}\left[\Delta F^{p}\right](x, \xi)\right| \\
& <\int_{0}^{s_{j}^{F}(x, \xi)}\left|\Sigma_{j}\left(M_{i j}\left(x, \xi, s_{j}\right)\right) \Delta F^{p}\left(M_{j}\left(x, \xi, s_{j}\right)\right)\right| d s
\end{aligned}
$$

Using (205) this yields

$$
\begin{aligned}
\left|\Delta F_{j}^{p+1}(x, \xi)\right| & <\bar{\Sigma} \int_{0}^{s_{j}^{F}(x, \xi)} \bar{f} M^{p} \\
& \frac{\left((\alpha+1)-\alpha x_{j}(x, \xi, s)-\xi_{j}(x, \xi, s)\right)^{p}}{p !} d s \\
& <\bar{f} \frac{\bar{\Sigma} M^{p}}{\delta} \frac{(-\alpha x-\xi+(\alpha+1))^{p+1}}{(p+1) !}
\end{aligned}
$$

which yields the result using (203)

Proof: We now prove the Initialization. Denoting

$$
\bar{f}=\max _{(x, \xi) \in \mathcal{T}_{0}} \max _{j=1 \ldots n+m}\left|f_{j}(x, \xi)\right|
$$

one has

$$
\forall j=1 \ldots n+m\left|\Delta F_{j}^{0}(x, \xi)\right|=\left|f_{j}\left(M_{j}^{0}(x, \xi)\right)\right|<\bar{f}
$$

Then, using Lemma 15, one can prove by recursion that

$$
\sum_{p=0}^{+\infty}\left|\Delta F^{p}(x, \xi)\right|<\bar{f} e^{M(-\alpha x-\xi+(\alpha+1))}
$$

Defining $F$ as

$$
F(x, \xi)=\sum_{p=0}^{+\infty} \Delta F^{p}(x, \xi)=\lim _{p \rightarrow \infty} F^{p}(x, \xi)
$$

and taking the limit $p \rightarrow \infty$ in (195) yields the result.

\section{REFERENCES}

[1] U. J. Aarsnes, Modeling of two-phase flow for estimation and control of drilling operations, Ph.D. thesis, Norwegian University of Science and Technology, 2016.

[2] U. J. F. Aarsnes, F. Di Meglio, S. Evje, and O.-M. Aamo, Controloriented drift-flux modeling of single and two-phase flow for drilling, Proceedings of the ASME 2014 Dynamic Systems and Control Conference, San Antonio, USA, October 22-24, 2014.

[3] U. J. F. Aarsnes, F. Di Meglio, R. Graham, and O. M. Aamo, A methodology for classifying operating regimes in underbalanced-drilling operations, SPE Journal 21 (2) (2015).

[4] Saurabh Amin, Falk M Hante, and Alexandre M Bayen, On stability of switched linear hyperbolic conservation laws with reflecting boundaries, Hybrid Systems: Computation and Control, Springer, 2008, pp. 602-605.

[5] Jean Auriol and Florent Di Meglio, Minimum time control of heterodirectional linear coupled hyperbolic pdes, Automatica 71 (2016), 300-307.

[6] _ Two sided boundary stabilization of two linear hyperbolic pdes in minimum time, https://hal-mines-paristech.archives-ouvertes.fr/hal01318708 (2016)
[7] G. Bastin and J.-M. Coron, On boundary feedback stabilization of non-uniform linear $2 \times 2$ hyperbolic systems over a bounded interval, Systems \& Control Letters 60 (2011), 900-906.

[8] G Bastin and JM Coron, Stability and boundary stabilization of 1-d hyperbolic systems, Preprint (2015).

[9] Haim Brezis, Functional analysis, sobolev spaces and partial differential equations, Springer Science \& Business Media, 2010.

[10] Jean-Michel Coron, Brigitte dAndréa Novel, and Georges Bastin, A lyapunov approach to control irrigation canals modeled by saint-venant equations, Proc. European Control Conference, Karlsruhe, 1999.

[11] Jean-Michel Coron, Long Hu, and Guillaume Olive, Stabilization and controllability of first-order integro-differential hyperbolic equations, arXiv preprint arXiv:1511.01078 (2015).

[12] Jean-Michel Coron, Rafael Vazquez, Miroslav Krstic, and Georges Bastin, Local exponential h^2 stabilization of a $2 \backslash$ times 2 quasilinear hyperbolic system using backstepping, SIAM Journal on Control and Optimization 51 (2013), no. 3, 2005-2035.

[13] Jonathan de Halleux, Christophe Prieur, J-M Coron, Brigitte d'Andréa Novel, and Georges Bastin, Boundary feedback control in networks of open channels, Automatica 39 (2003), no. 8, 1365-1376.

[14] F. Di Meglio, D. Bresch-Pietri, and U. J. F. Aarsnes, An adaptive observer for hyperbolic systems with application to underbalanced drilling, Proceeding of the 2014 IFAC World Congress, 2014, pp. 11391-11397.

[15] Florent Di Meglio, Dynamics and control of slugging in oil production, $\mathrm{Ph} . D$. thesis, Ecole Nationale Supérieure des Mines de Paris, 2011.

[16] Florent Di Meglio, Miroslav Krstic, and Rafael Vazquez, A backstepping boundary observer for a class of linear first-order hyperbolic systems, Control Conference (ECC), 2013 European, IEEE, 2013, pp. 1597-1602.

[17] Stéphane Dudret, Karine Beauchard, Fouad Ammouri, and Pierre Rouchon, Stability and asymptotic observers of binary distillation processes described by nonlinear convection/diffusion models, American Control Conference (ACC), 2012, IEEE, 2012, pp. 3352-3358.

[18] Long Hu, Florent Di Meglio, Rafael Vazquez, and Miroslav Krstic, Control of homodirectional and general heterodirectional linear coupled hyperbolic pdes, (2015).

[19] Fritz John, Continuous dependence on data for solutions of partial differential equations with a prescribed bound, Communications on pure and applied mathematics 13 (1960), no. 4, 551-585.

[20] Tatsien Li and Bopeng Rao, Strong (weak) exact controllability and strong (weak) exact observability for quasilinear hyperbolic systems, Chinese Annals of Mathematics, Series B 31 (2010), no. 5, 723-742.

[21] Rafael Vazquez, Jean-Michel Coron, Miroslav Krstic, and Georges Bastin, Local exponential h 2 stabilization of a $2 \times 2$ quasilinear hyperbolic system using backstepping, Decision and Control and European Control Conference (CDC-ECC), 2011 50th IEEE Conference on, IEEE, 2011, pp. 1329-1334.

[22] Rafael Vazquez and Miroslav Krstic, Bilateral boundary control of one-dimensional first-and second-order pdes using infinite-dimensional backstepping, arXiv preprint arXiv:1603.04913 (2016).

[23] Gerald Beresford Whitham, Linear and nonlinear waves, vol. 42, John Wiley \& Sons, 2011.

[24] Cheng-Zhong Xu and Gauthier Sallet, Exponential stability and transfer functions of processes governed by symmetric hyperbolic systems, ESAIM: Control, Optimisation and Calculus of Variations 7 (2002), 421-442.

Jean Auriol Jean Auriol is a PhD student at Centre Automatique et Systmes of MINES ParisTech, part of PSL Research University, under the direction of Florent Di Meglio. His current research interests include control and estimation design for hyperbolic PDEs

Florent Di Meglio Florent Di Meglio is tenured professor at the Centre Automatique et Systmes of MINES ParisTech, part of PSL Research University. He received his Ph.D. from the same university in Mathematics and Control in 2011, and was a Posdoctoral Researcher at UC San Diego from 2011 to 2012. His current research interests include control and estimation design for hyperbolic PDEs, with application to process control, most notably multiphase flow controland oildrilling. 